\title{
DNA Scaffolds for the Dictated Assembly of Left-/Right-Handed Plasmonic AuNP Helices with Programmed Chiro-Optical Properties
}

\author{
Alessandro Cecconello, ${ }^{\dagger}$ Jason S. Kahn, ${ }^{\dagger}$ Chun-Hua Lu, ${ }^{\dagger}$ Larousse Khosravi Khorashad, \\ Alexander O. Govorov ${ }^{\dagger}$ and Itamar Willner ${ }^{\dagger} *$ \\ ${ }^{\dagger}$ The Institute of Chemistry, The Center for Nanoscience and Nanotechnology, The Hebrew \\ University of Jerusalem, Jerusalem 91904, Israel. \\ ${ }^{\ddagger}$ Department of Physics and Astronomy, Ohio University, Athens, Ohio 45701, USA.
}

Supporting Information 


\section{Materials}

Sodium cloride, Magnesium acetate, Bis( $p$-sulfonatophenyl)phenylphosphine dihydrate dipotassium salt, and Tris(2-carboxyethyl)phosphine hydrochloride were purchased from Sigma-Aldrich. MetaPhor agarose was purchased from Lonza (USA). Snakeskin 10kDa dialysis tube was purchased from Thermo Scientific (USA). Gold colloid (AuNP) $10 \mathrm{~nm}$ was purchased from British Biocell (BBI Solutions). Amicon ultra $100 \mathrm{kDa}$ and $30 \mathrm{kDa}$ filtering tubes were purchased from Millipore. Disposable filter units were purchased from Whateman GmbH. All DNA oligonucleotides were purchased from Integrated DNA Technologies. DNA sequences for the quasi-ring strands and thiolated strands were HPLC-purified, all other sequences were ordered as desalted material. Ultrapure water from a NANOpure Diamond (Barnstead) source was used in all experiments. Tris Acetate EDTA buffer (TAE) 50X and Tris Borate EDTA (TBE) buffer $5 \mathrm{X}$ were purchased from Biological Industries (Israel).

\section{Instruments}

Dynamic light scattering (DLS) experiments were performed with a Malvern Zetasizer Nano. High resolution transmission electron microscopy images were taken with a MagellanT XHR SEM (FEI). Circular dichroism spectra were obtained with a Jasco J-810 spectropolarimeter. Atomic force microscopy (AFM) characterization was performed with a Bruker Multimode $\mathrm{V}$ and ScanAsyst-Air or ScanAsyst-Fluid+ probes. UV2401PC Shimadzu spectrophotometer was used to collect absorbance data. Thermostatic bath Heto CBN 8-30, thermal cycler MultiGene Labnet International Inc.

\section{Methods}

\subsection{Sequence Design}

Initial design of the barrel sequences was carried out by using CadNano2 software (http://cadnano.org/blog/cadnano-version-220), following a reported architecture. ${ }^{1}$ Positioning of the promoting strands (Ps) and tethers (T) for the AuNPs, was achieved taking into consideration that (1) each quasi-ring is carrying one T for a total of 12 AuNP tethers per barrel; (2) the strands $\mathbf{T}$ must be at a distance of $16 \mathrm{bp}$ moving from one quasi-ring position to the next; (3) the

positioning of the tethers $\mathbf{T}$ in the barrels $\mathrm{BT}_{1,2} \mathrm{~L}$ and $\mathrm{BT}_{1,2} \mathrm{R}$ is the mirror image of the 
positioning of the tethers in barrels $\mathrm{BT}_{3,4} \mathrm{~L}$ and $\mathrm{BT}_{3,4} \mathrm{R}$; (4) positioning of the sequences Ps followed the idea that a staple sequence, at the crossover junction, must be pointing parallel the surface of the barrel and, following DNA duplex geometry, the 8th bp downhill the crossover junction must be pointing at an angle of $270^{\circ}$ in respect to the surface of the barrel; (5) Ps sequences at the $3^{\prime}$ of the staple where the crossover is facing the upper quasi-ring are pointing out of the plane of the sheet while Ps sequences at the $3^{\prime}$ of the staple where the crossover is facing the lower quasi-ring are pointing inside the plane of the sheet (see Figures 1(D) and S2), while the opposite happens when the Ps elongating sequence is positioned at the 5 ' of the staple strand; (6) Ps and $\mathbf{T}$ sequences are positioned far away to avoid steric and/or electrostatic repulsion between Ps strands and the AuNPs and thus ensure high yields for the hybridization of the DNA modified AuNPs on the barrels; (7) special care was used to design the sequences joining the two quasi-rings R5-R5. This was due to the fact that the AuNP tethers T must follow, once the barrel is formed, a full helix without defects. In order to accomplish this geometrical constraint, point (2) of this list must be applied carefully.

\subsection{Barrels and Nanotubes Formation}

The DNA nanotubes were formed by subjecting a $50 \mu \mathrm{l}$ aqueous solution containing $100 \mathrm{nM}$ of each quasi-ring sequence and 500nM of each staple (see Table 2 for the list of specific staple sequences), $15 \mathrm{mM} \mathrm{Mg}(\mathrm{Ac})_{2}$, and 1 XTAE buffer to a $95^{\circ}$ for 5 minutes and then slowly decreasing the temperature to $20^{\circ}$ in a thermostatic bath for 24 hours. The DNA barrels were formed by subjecting a $50 \mu \mathrm{l}$ aqueous solution containing $100 \mathrm{nM}$ of each quasi-ring sequence and 100nM of each staple (see Table 3, 4, 5, and 6 for the list of specific staple sequences), 20 $\mathrm{mM} \mathrm{Mg}(\mathrm{Ac})_{2}$, and 1 XTAE buffer to a $90^{\circ}$ and decreasing the temperature to $60^{\circ}$ within 20 minutes, followed by a 14 hours further decrease in temperature to $4^{\circ}$ in a thermal cycler.

\subsection{AFM Characterization and DLS Measurements}

The DNA nanotubes solution was deposited on a freshly cleaved mica support. After 10' minutes the surface was washed with water and dried. Imaging was performed in air, using the Bruker ScanAsyst software and the appropriate probe (ScanAsyst-Air), Figure S1.

The DNA barrel solution was filtered twice using an Amicon $30 \mathrm{kDa} \mathrm{MWCO}$ at $10000 \mathrm{~g}$ for 5 minutes at $10^{\circ} \mathrm{C}$, using the buffer employed in the assembly of the barrels. AFM imaging was 
conducted in liquid, using the same buffer. Few microliters of the sample were deposited on freshly cleaved mica, subsequently the mica surface was washed two times with buffer, and then subjected to a buffer droplet while imaging the surface in the solution. Bruker ScanAsyst software was used with the appropriate ScanAsyst-Fluid+ probe.

Due to the very low scattering efficiency of the DNA barrel structure, all solutions used for the DLS analysis were filtered with a $0.22 \mu \mathrm{m}$ disposable filter and all vessels and cuvettes were carefully rinsed to avoid the presence of dust and small particles. $300 \mu \mathrm{l}$ of barrel solution was used at the concentration of $100 \mathrm{nM}$. A value of 2.0 for the refraction coefficient was used and the results showed a good correlation level between the elaborated size peak and the scattering signal.

\subsection{AuNP Modification and Their Application to the Barrels}

A published method was followed for the AuNPs modification. ${ }^{2}$

1- 10nm AuNPs were incubated for 8 hours with an excess of $\operatorname{bis}(p$ sulfonatophenyl)phenylphosphine dihydrate dipotassium salt and then filtered using Amicon Ultra $100 \mathrm{kDa}$ MWCO device at $5000 \mathrm{~g}$ for 3 minutes, at room temperature, to eliminate the excess of capping and concentrate the particles. The absorbance at $\lambda=520 \mathrm{~nm}$ and the AuNP extinction coefficient was used to estimate the concentration of the AuNPs. The particles protected with the phosphine capping were used as starting material for the DNA functionalization.

2- Thiolated DNA sequences $\mathbf{T}_{\mathbf{1}, \mathbf{3}^{\prime}}$ and $\mathbf{T}_{\mathbf{2}, \mathbf{4}^{\prime}}(15 \mu \mathrm{M})$ were incubated at room temperature for 1 hour with tris(2-carboxyethyl)phosphine hydrochloride $(15 \mathrm{mM})$ and then added to a AuNP solution containing $100 \mathrm{mM} \mathrm{NaCl}, \quad 0.5 \mathrm{XTBE} \quad$ buffer, $0.5 \quad \mathrm{mg} / \mathrm{ml} \quad \operatorname{bis}(p$ sulfonatophenyl)phenylphosphine dihydrate dipotassium salt. The ratio DNA:AuNPs was equal to 1:1. Furthermore, DNA sequences partially complementary to $\mathbf{T}_{\mathbf{1}, \mathbf{3}^{\prime}}$ and $\mathbf{T}_{\mathbf{2}, 4^{\prime}}\left(\mathbf{T}_{\mathbf{1}, 3^{\prime \prime}}\right.$ and $\left.\mathbf{T}_{\mathbf{2}, 4^{\prime \prime}}\right)$ were added to the respective AuNP solution, in order to elongate the total length of the DNA strand attached to the particle and facilitate the following separation step. This solution was incubated for three hours at room temperature. 
3- A 3\% MetaPhor agarose gel in 0.5XTBE buffer was prepared in a gel electrophoresis bath. The AuNPs functionalized in step 2 were added to the gel electrophoresis wells and a $10 \mathrm{~nm}$ AuNP reference (non-functionalized particles) was loaded in a different well. The samples ran for 2 hours at 80 volts.

4- When sufficient separation of the DNA-functionalized particles was obtained, the band corresponding to the single-nucleic acid modified AuNPs was excised (the first band with larger hydrodynamic volume in respect to the reference AuNPs). The excised band was inserted in a $10 \mathrm{kDa}$ MWCO dialysis tube with $5 \mathrm{ml}$ of $0.5 \mathrm{XTBE}$ buffer and the tube was then sealed. To extract the particles from the agarose band the dialysis tube containing the excised band was placed in a electrophoresis bath and a voltage equals to $150 \mathrm{~V}$ was applied. The modified particles were then extracted from the gel band and the solution containing the particles was collected.

5- A $0.2 \mu \mathrm{m}$ filter was used to filter the solution collected from the dialysis tube to eliminate gel pieces. The particles were then mixed with a short thiolated DNA oligonucleotide (Stab) and releasing DNA strands ( $\mathbf{R T}_{1,3}{ }^{\prime \prime}$ and $\mathbf{R} \mathbf{T}_{2,4} "$ ) that remove the nucleic acids $\mathbf{T}_{1,3}$ " and $\mathbf{T}_{2,4}$ " from the functionalized particles. The samples were incubated at room temperature for 48 hours, prior to filtering using Amicon Ultra 100kDA MWCO to eliminate the excess of $\mathrm{HS}^{-\mathrm{dT}_{5}}$ (Stab) and $\mathbf{R T}_{1,3} \mathbf{3}^{\prime \prime} \mathbf{T}_{\mathbf{1}, 3}$ " and $\mathbf{R} \mathbf{T}_{2,4}{ }^{\prime \prime} / \mathbf{T}_{\mathbf{2}, 4} "$ duplexes.

6- To obtain the AuNP helices, $3 \mu \mathrm{L}$ of the assembled barrel solution was mixed with the appropriate mix of $\mathbf{T}^{\prime}$-modified Au NPs, with a ratio 1:12, in a solution containing $20 \mathrm{mM}$ magnesium acetate in $0.5 \mathrm{XTAE}$ buffer for 48 hours at room temperature. The solution was then diluted in the same buffer to a volume of $120 \mu \mathrm{l}$ ( $1 \mathrm{nM}$ concentration of the helices) immediately followed by $\mathrm{CD}$ and TEM characterization.

\subsection{CD and TEM Characterization of the AuNP Helices}

The samples were analyzed in a Jasco CD spectropolarimeter in a $1 \mathrm{~cm}$ light-path quartz cuvette and the CD spectra were corrected with a blank (20mM magnesium acetate in $0.5 \mathrm{XTAE})$. Three 
signal accumulations for each experiment were averaged and the final spectra were smoothed. TEM characterization of the Au NP assemblies was performed with XHR-STEM Magellan (FEI). Few microliters of the Au NP-decorated barrels solution ( $1 \mathrm{nM})$ were deposited on a TEM copper mesh and freeze-dried prior to imaging.

\subsection{Anisotropy g-factor measurements}

Anisotropy g-factors for the for chiroplasmonic structures $\mathrm{BT}_{1,2} \mathrm{~L}, \mathrm{BT}_{1,2} \mathrm{R}, \mathrm{BT}_{3,4} \mathrm{~L}$, and $\mathrm{BT}_{3,4} \mathrm{R}$ were obtained according to the formula $\mathrm{g}=\Delta \varepsilon / \varepsilon$ where $\Delta \varepsilon$ and $\varepsilon$ are molar circular dichroism and molar extinction coefficient, respectively. Using the CD spectra and the absorbance spectra of the different structures, the associated g-factors were calculated.

\subsection{Computational modeling of the CD spectra}

We employed discrete dipole approximation method ${ }^{3-5}$ (DDA) for our theoretical understanding of the measured CD signals obtained from left and right handed helical structures. The frequency dependent bulk permittivity of gold has been taken from the literature. ${ }^{6}$ The dielectric constant of the medium is taken as 1.8 (water and DNAs) to account for the surrounding medium of the helical structures. In the optical experiments, the structures are randomly oriented in solution. Therefore, in order to calculate the CD signal, one requires to take the average of the $\mathrm{CD}$ for all possible directions of the incident left and right circularly polarized (LCP/RCP) lights on the structures. To reduce time consuming computations while achieving accurate results, in the simulation, LCP and RCP beams are sent to the structure from three orthogonal directions. It was previously demonstrated that this approach is adequate to compute the features of small structures. ${ }^{7}$ Accordingly, the extinction cross sections for circularly-polarized photons are calculated. Subsequently, for each orthogonal direction, the CD signal is obtained by the difference between the LCP and RCP extinction cross sections. Finally, the averaged CD was computed by taking the average of the three directional CD signals. The input parameters for our optical plasmonic modes were the coordinates of NPs. 

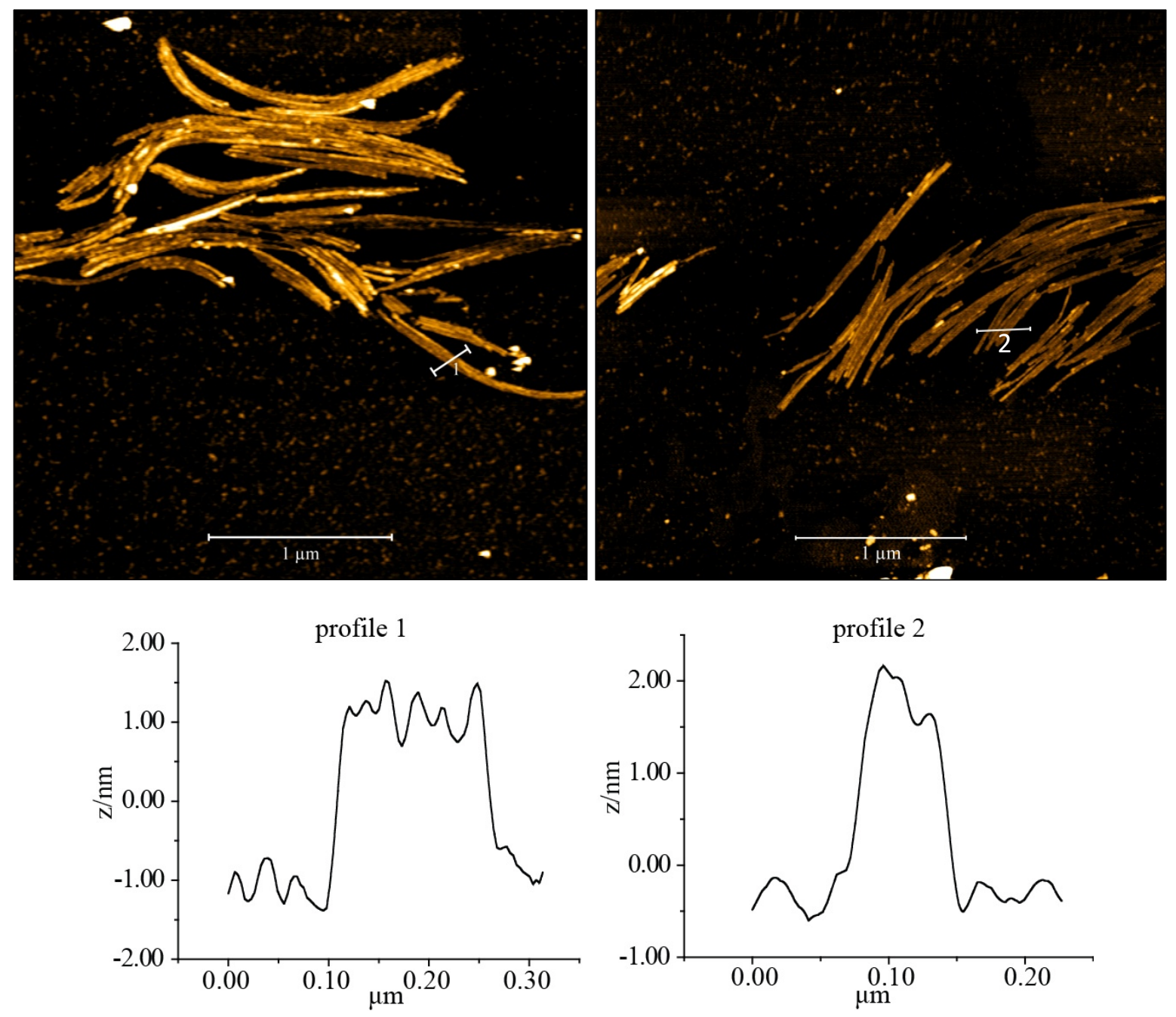

Figure S1. AFM characterization of the DNA nanotubes assembled by stacking the DNA barrels in a head-tail configuration. It should be noted that the nanotubes tend to form bundles during the sample deposition and the drying process. 
Extension of the staple strands:

promoter strands Ps $=$ tethers $T$

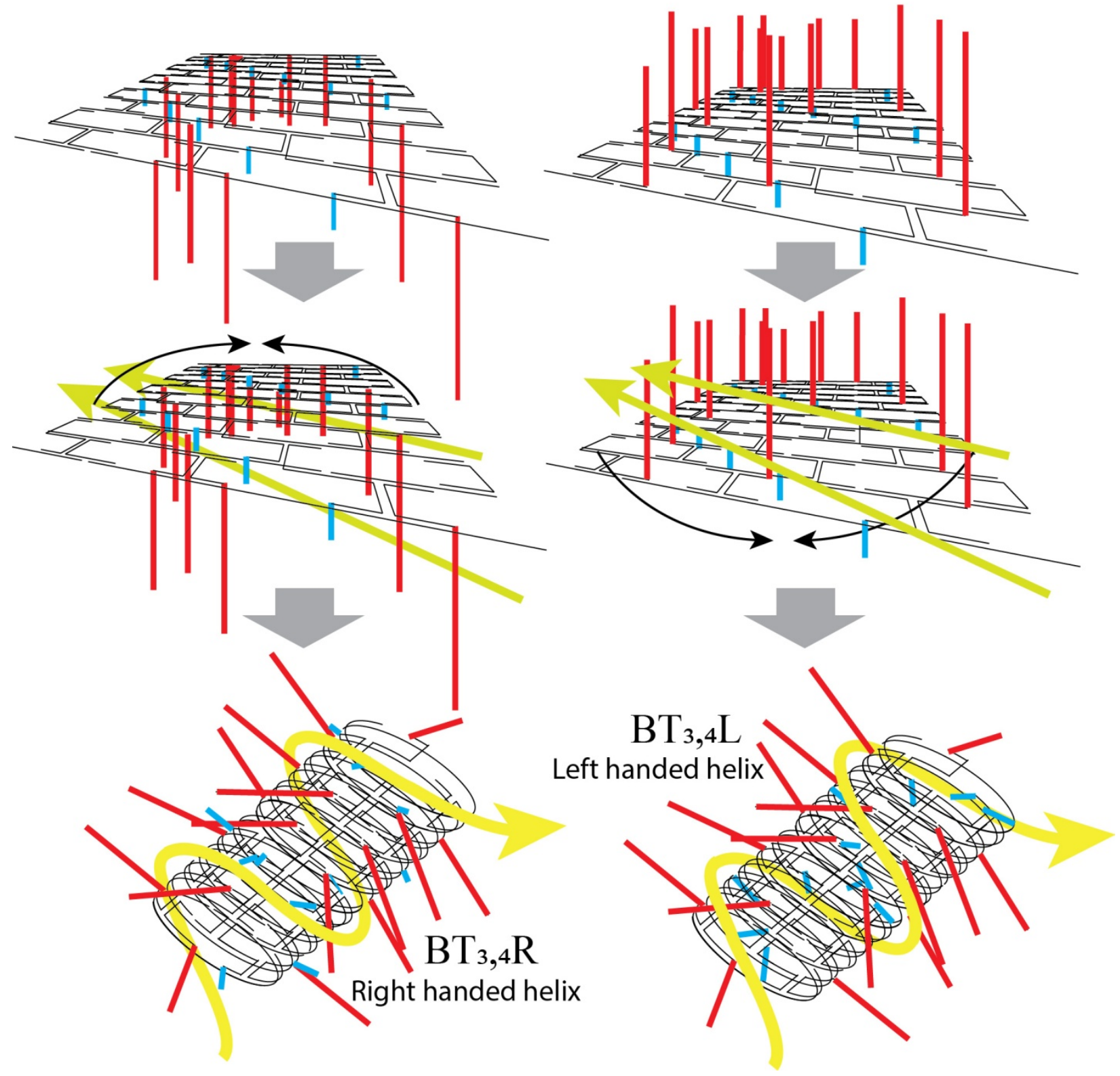

Figure S2. Schematics of the folding and structures of DNA nanobarrels $\mathrm{BT}_{3,4} \mathrm{R}$ and $\mathrm{BT}_{3,4} \mathrm{~L}$. 


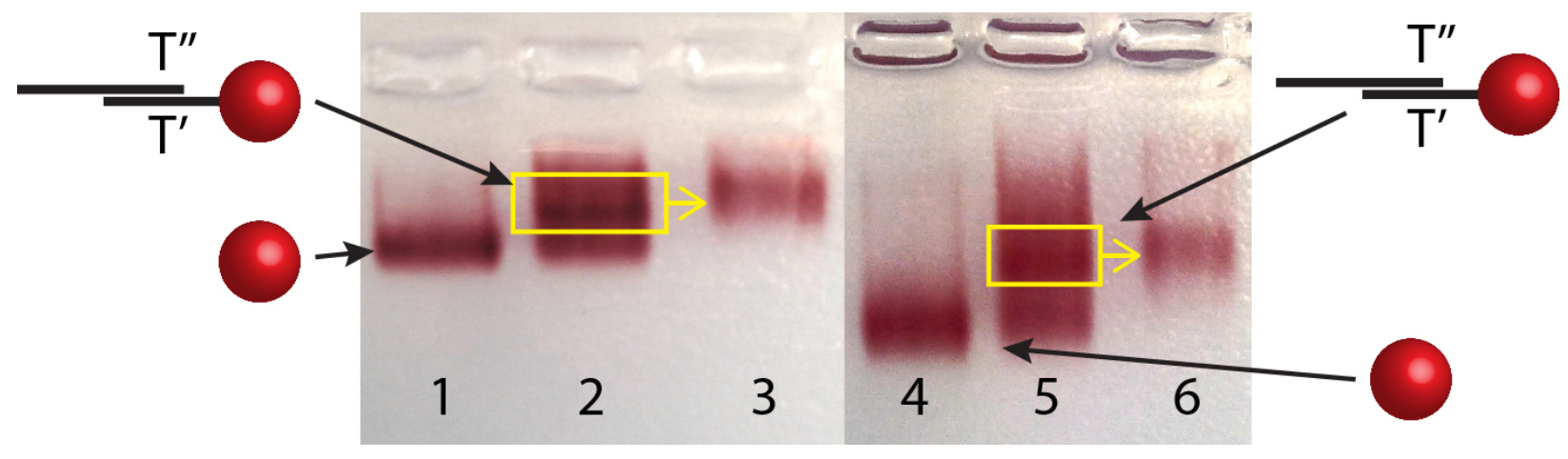

Figure S3. Single-nucleic acid modified 10nm AuNPs separation in agarose gel electrophoresis. Lanes: 1- Reference 10nm AuNPs; 2- $\mathbf{T}_{\mathbf{1 , 3}}$-modified 10nm AuNPs; 3- single-nucleic acid $\mathbf{T}_{\mathbf{1 , 3}} \mathbf{3}^{-}$ modified 10nm AuNPs after gel electrophoresis separation and extraction from the agarose gel; 4- reference 10nm AuNPs; 5- $\mathbf{T}_{\mathbf{2}, \mathbf{4}}$-modified 10nm AuNPs; 6- single-nucleic acid $\mathbf{T}_{\mathbf{2}, \mathbf{4}}$-modified $10 \mathrm{~nm}$ AuNPs after gel electrophoresis separation and extraction from the agarose gel. The yellow squares highlight the excised band containing single-nucleic acid modified AuNPs that were loaded on the right side lane (lanes 3 and 6) to compare the single-modified AuNPs with the mixture containing unmodified particles, single-modified particles and multi-modified particles (lanes 2 and 5). 

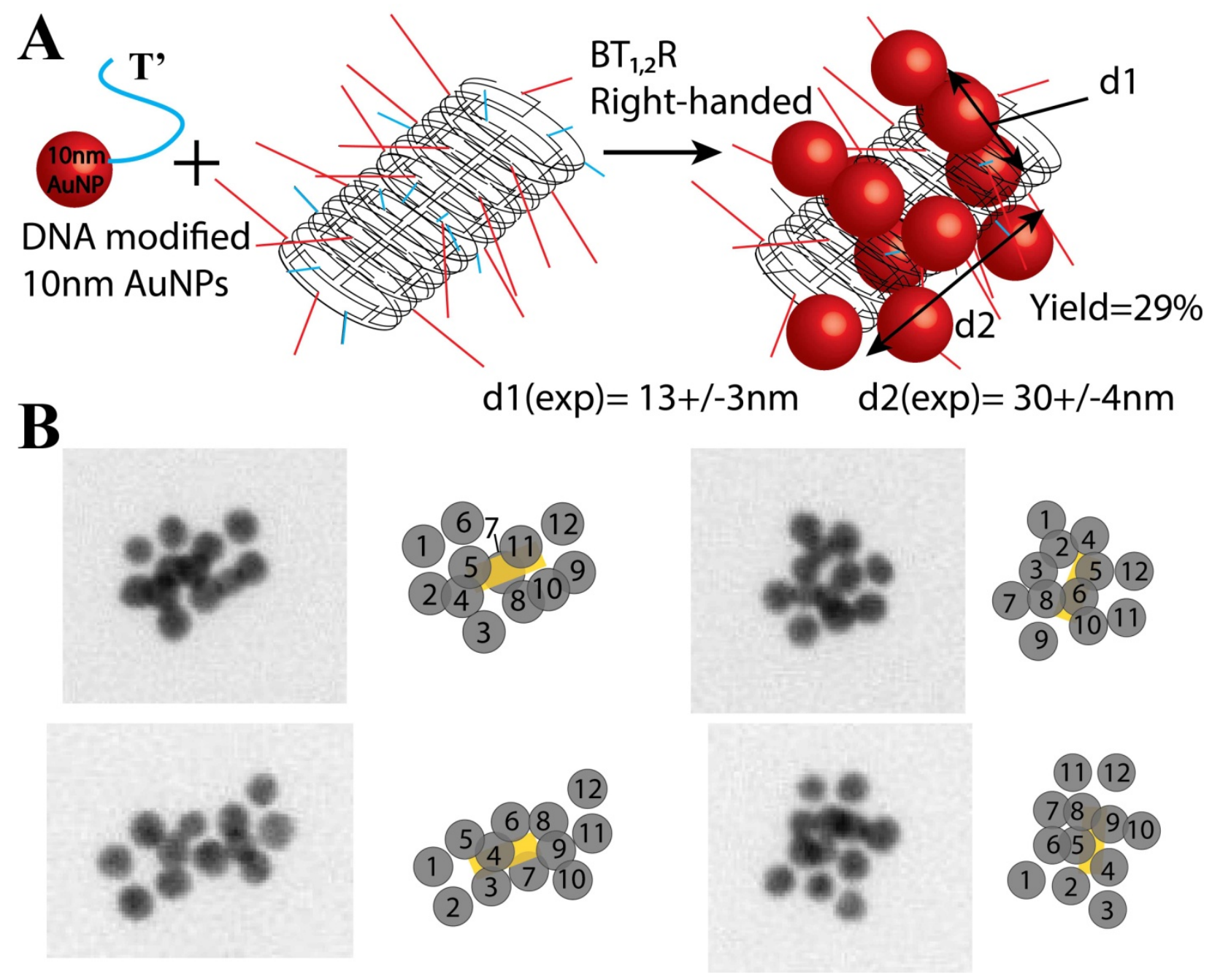

Figure S4. A. Scheme of the twelve $10 \mathrm{~nm}$ AuNPs helical assembly decorating the DNA nanobarrel $\mathrm{BT}_{1,2} \mathrm{R}$. B. Examples of HR-STEM pictures of the AuNP helices on the $\mathrm{BT}_{1,2} \mathrm{R}$ scaffold. On the right side of each high magnification HR-STEM picture is a scheme of the AuNP assembly where each particle is numbered from 1 to 12 . 

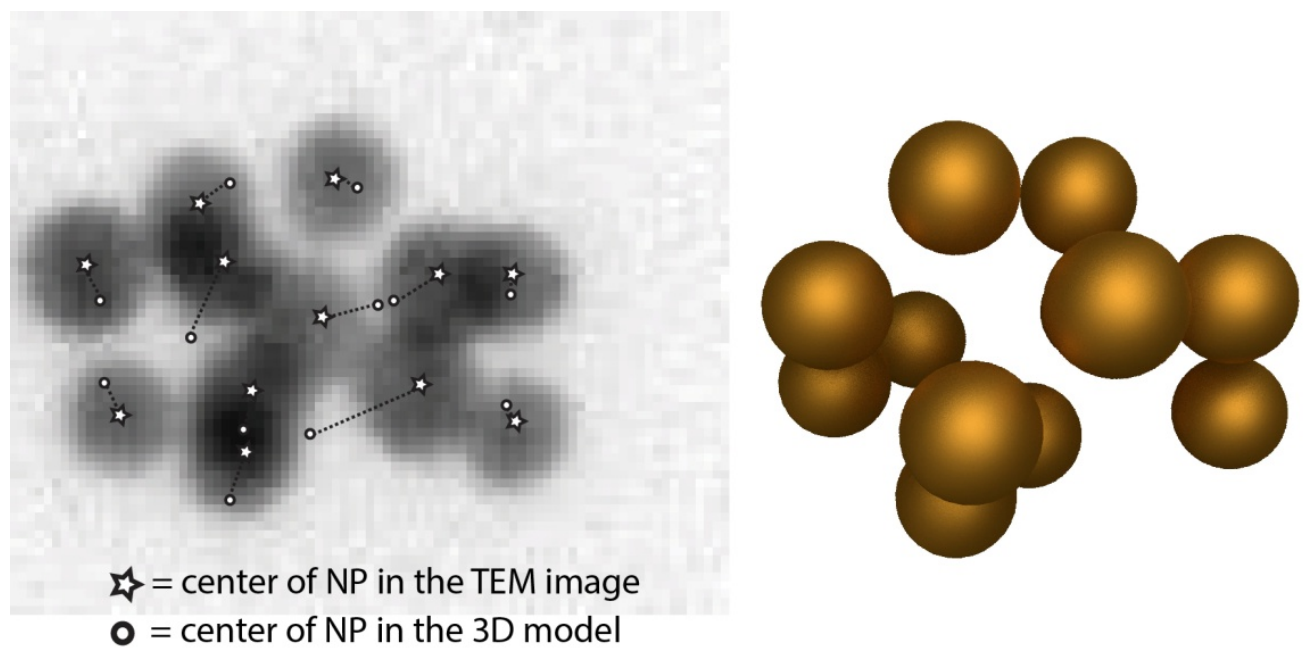

Figure S5a. Center-to-center distances comparison between the 3D model of the left-hand particle helix and the left-hand AuNP helix STEM image.
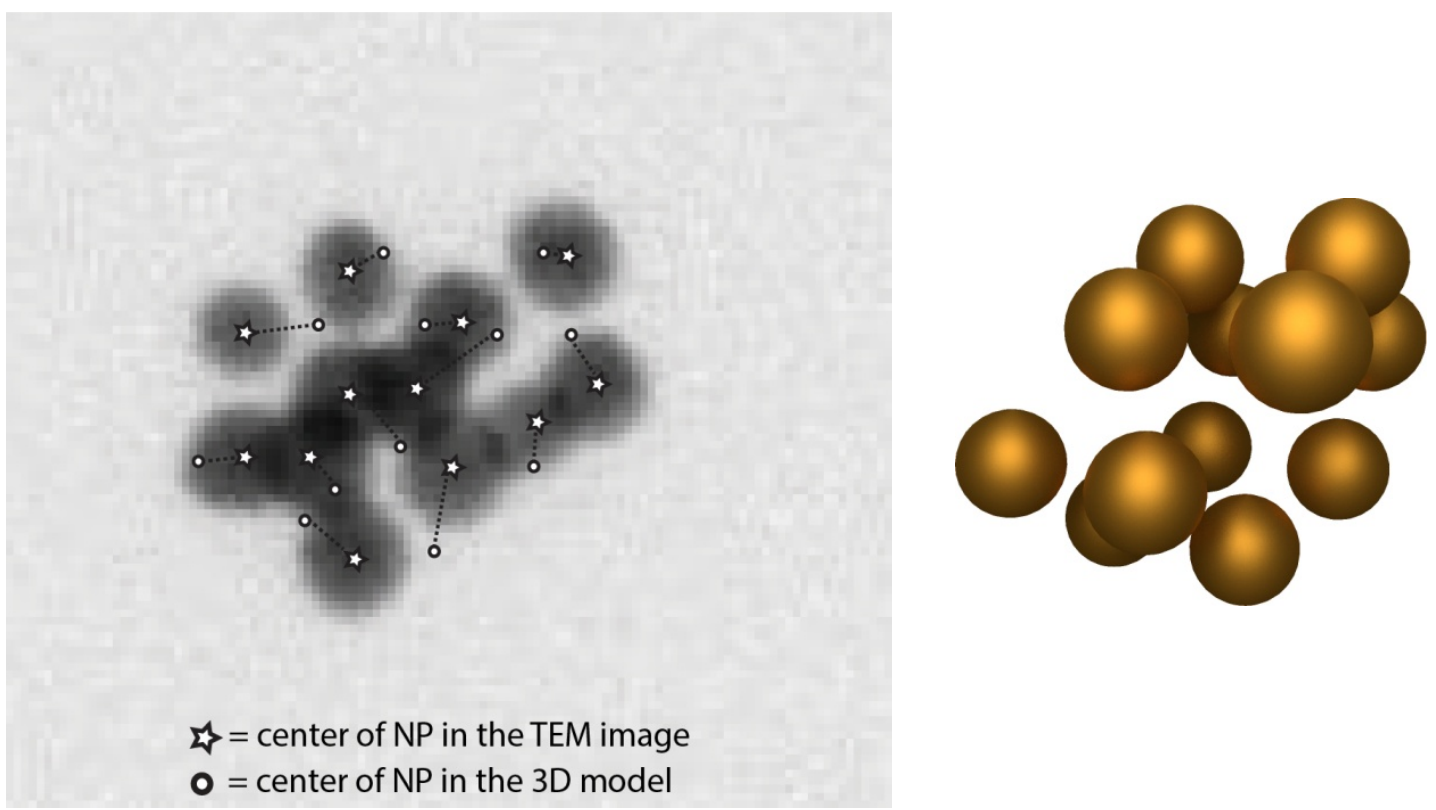

Figure S5b. Center-to-center distances comparison between the 3D model of the right-hand particle helix and the right-hand AuNP helix STEM image.

Figure S5 relates the two-dimensional HR-TEM images of the 12 AuNP structures associated with $\mathrm{BT}_{1,2} \mathrm{~L}$ (a) and $\mathrm{BT}_{1,2} \mathrm{R}$ (b) with the two-dimensional projection of the $3 \mathrm{D}$ model of the leftand right-handed helical structures of the NPs (the three-dimensional model provided in Figure S11, used to calculate the CD spectra). Although the drying of the chiral AuNP structures for the HR-TEM might distort the helical patterns of the NPs, we find that the structural features of the two-dimensional HR-TEM images of the AuNPs associated with $\mathrm{BT}_{1,2} \mathrm{~L}$ (a) and $\mathrm{BT}_{1,2} \mathrm{R}(\mathrm{b})$ fit 
well with the expected two-dimensional projection of the calculated 3D helical structures of the AuNPs. Figure S4a depicts the super-position of the configuration of the 2D projected model of $\mathrm{BT}_{1,2} \mathrm{~L}$ on the experimental HR-TEM. The average center-to-center distance corresponds to $4.2 \pm 2.5 \mathrm{~nm}$. Similarly, Figure S4b shows the super-position of the $2 \mathrm{D}$ projected model of the $\mathrm{BT}_{1,2} \mathrm{R}$ on the experimental HR-TEM. The average inter-particle distance corresponds to $4.8 \pm 1.9$ $\mathrm{nm}$.
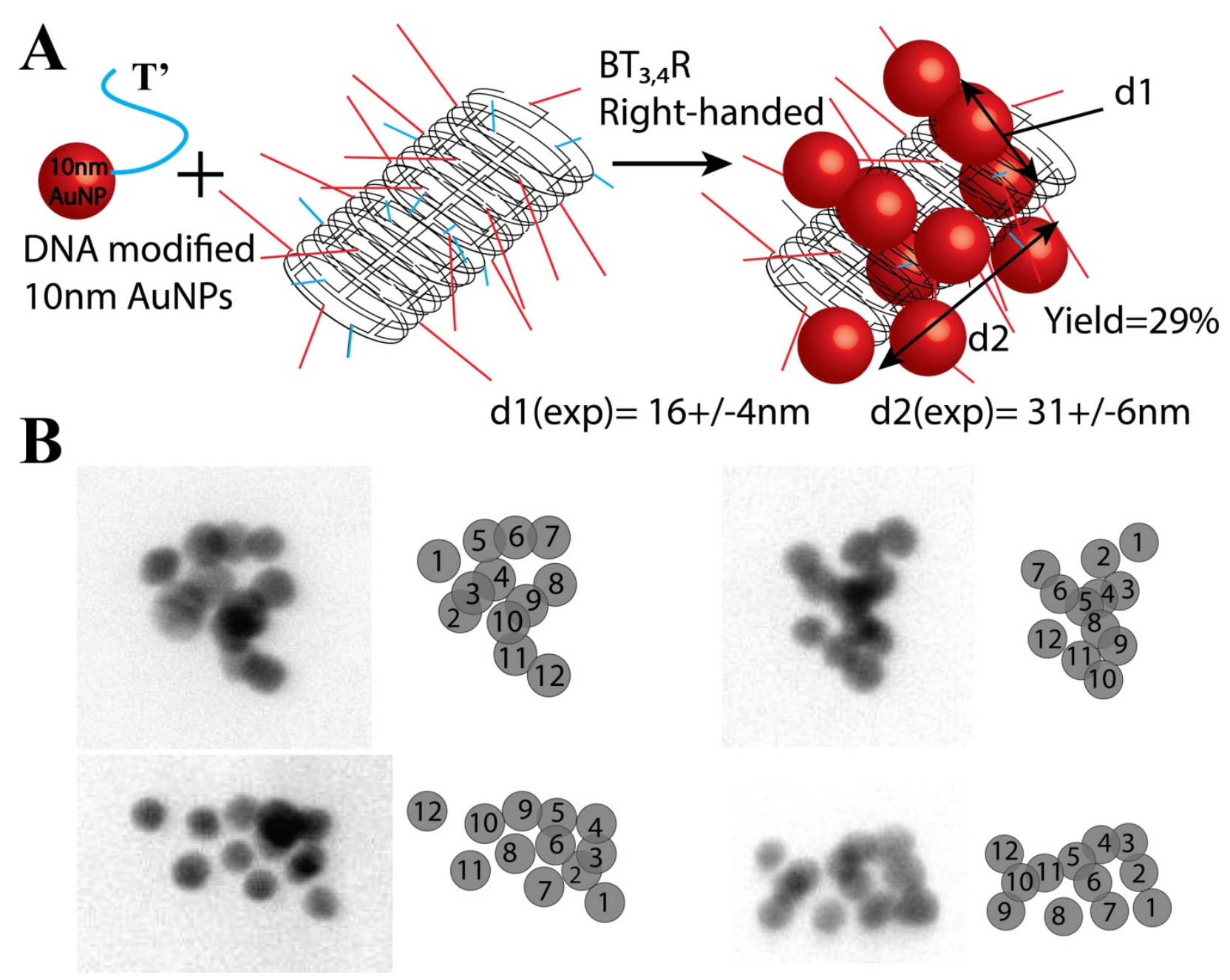

Figure S6. A. Scheme of the twelve $10 \mathrm{~nm}$ AuNPs helical assembly decorating the DNA nanobarrel $\mathrm{BT}_{3,4} \mathrm{R}$. B. Examples of HR-STEM pictures of the AuNP helices on the $\mathrm{BT}_{3,4} \mathrm{R}$ scaffold. On the right side of each high magnification HR-STEM picture is a scheme of the AuNP assembly where each particle is numbered from 1 to 12 . 

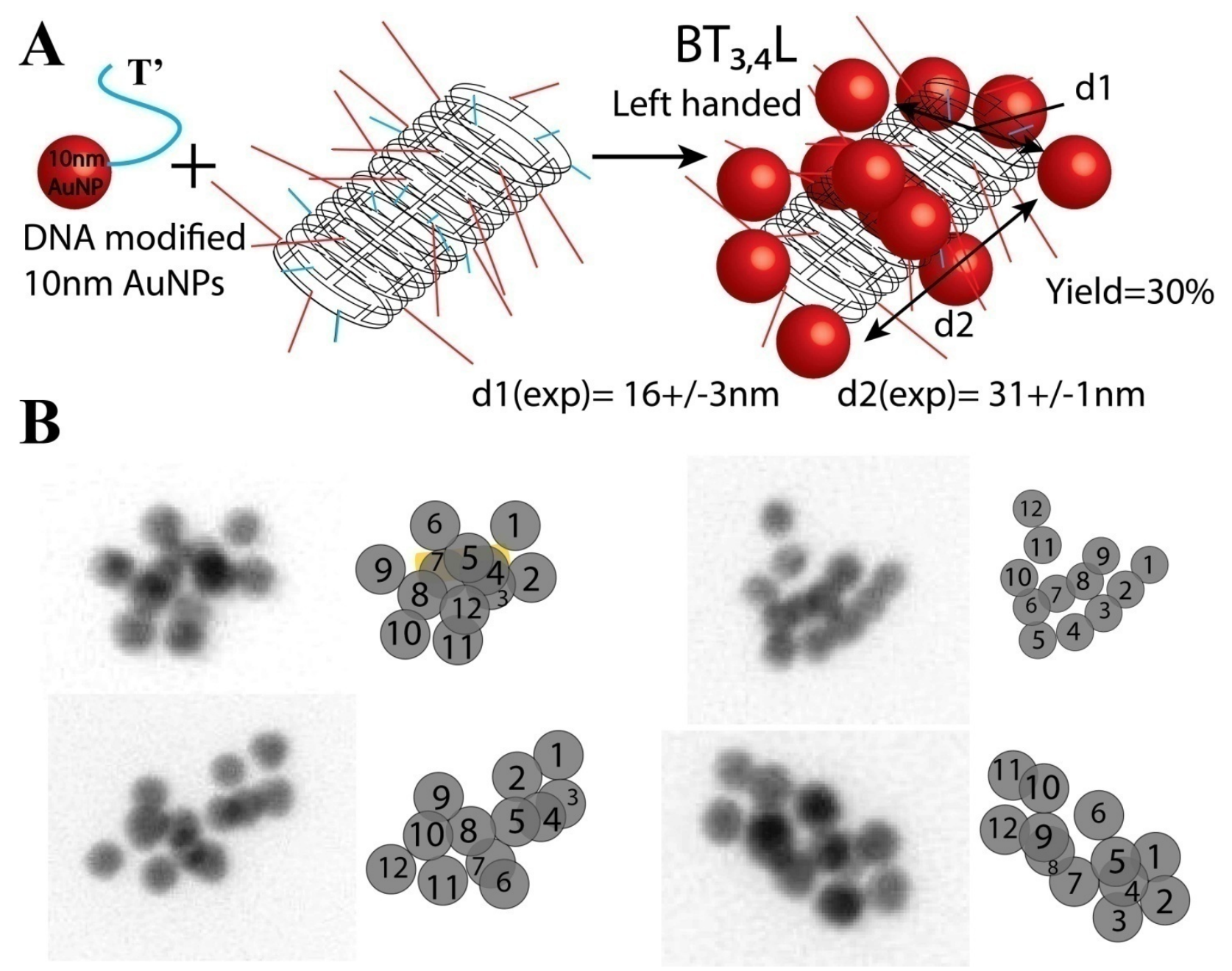

Figure S7. A. Scheme of the twelve $10 \mathrm{~nm}$ AuNPs helical assembly decorating the DNA nanobarrel $\mathrm{BT}_{3,4} \mathrm{~L}$. B. Examples of HR-STEM pictures of the AuNP helices on the $\mathrm{BT}_{3,4} \mathrm{~L}$ scaffold. On the right side of each high magnification HR-STEM picture is a scheme of the AuNP assembly where each particle is numbered from 1 to 12 . 


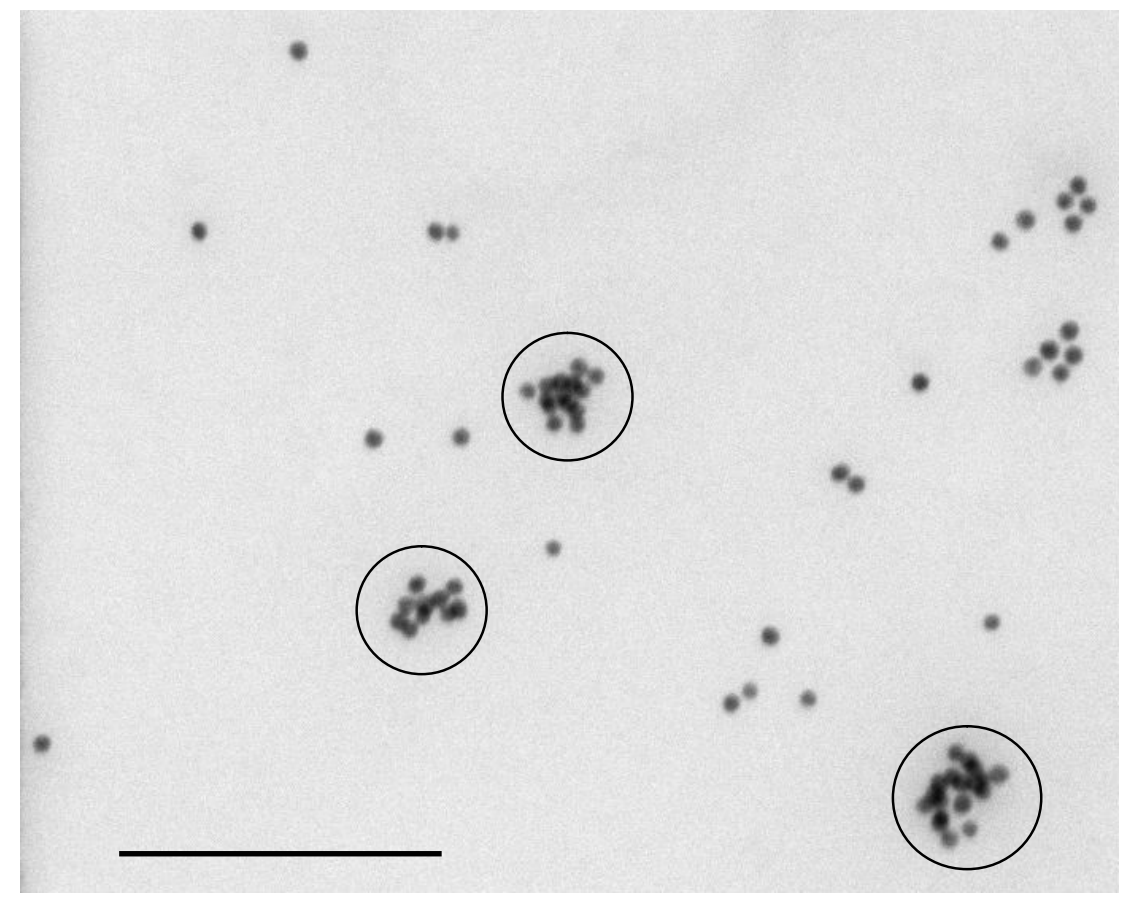

Figure S8a. Example of large area HRSTEM picture of the twelve $10 \mathrm{~nm}$ AuNP helical assembly $\mathrm{BT}_{1,2} \mathrm{~L}$ (bar=150nm).

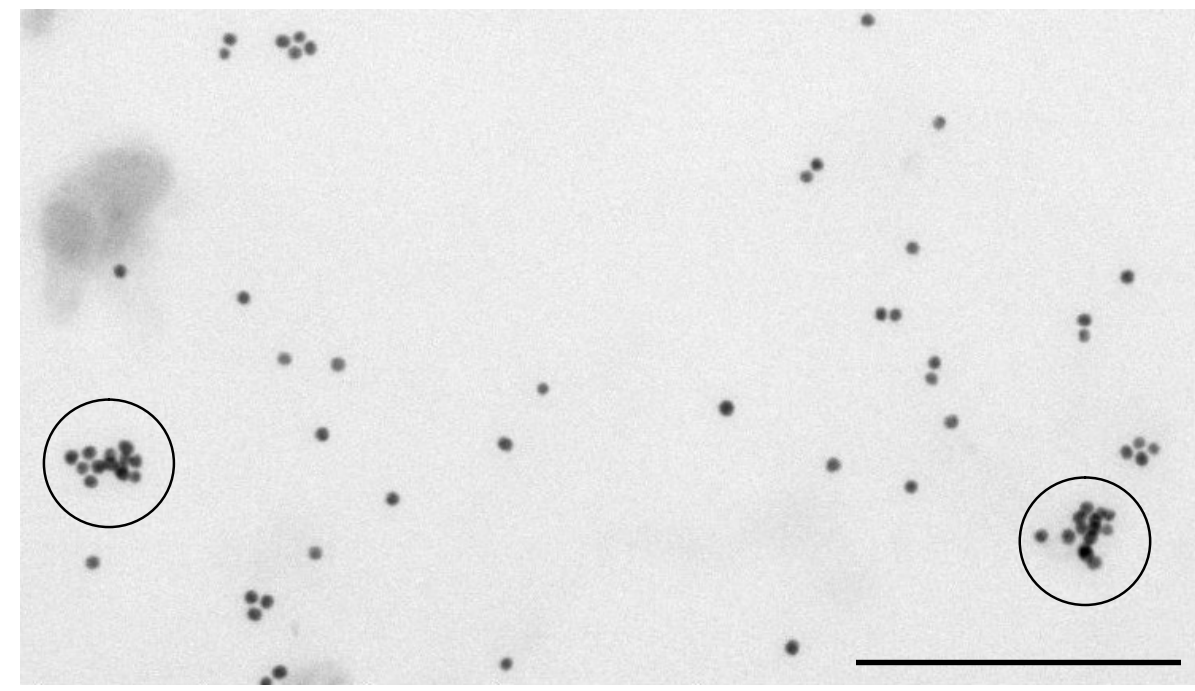

Figure S8b. Example of large area HRSTEM picture of the twelve $10 \mathrm{~nm}$ AuNP helical assembly $\mathrm{BT}_{1,2} \mathrm{~L}$ (bar $\left.=200 \mathrm{~nm}\right)$. 


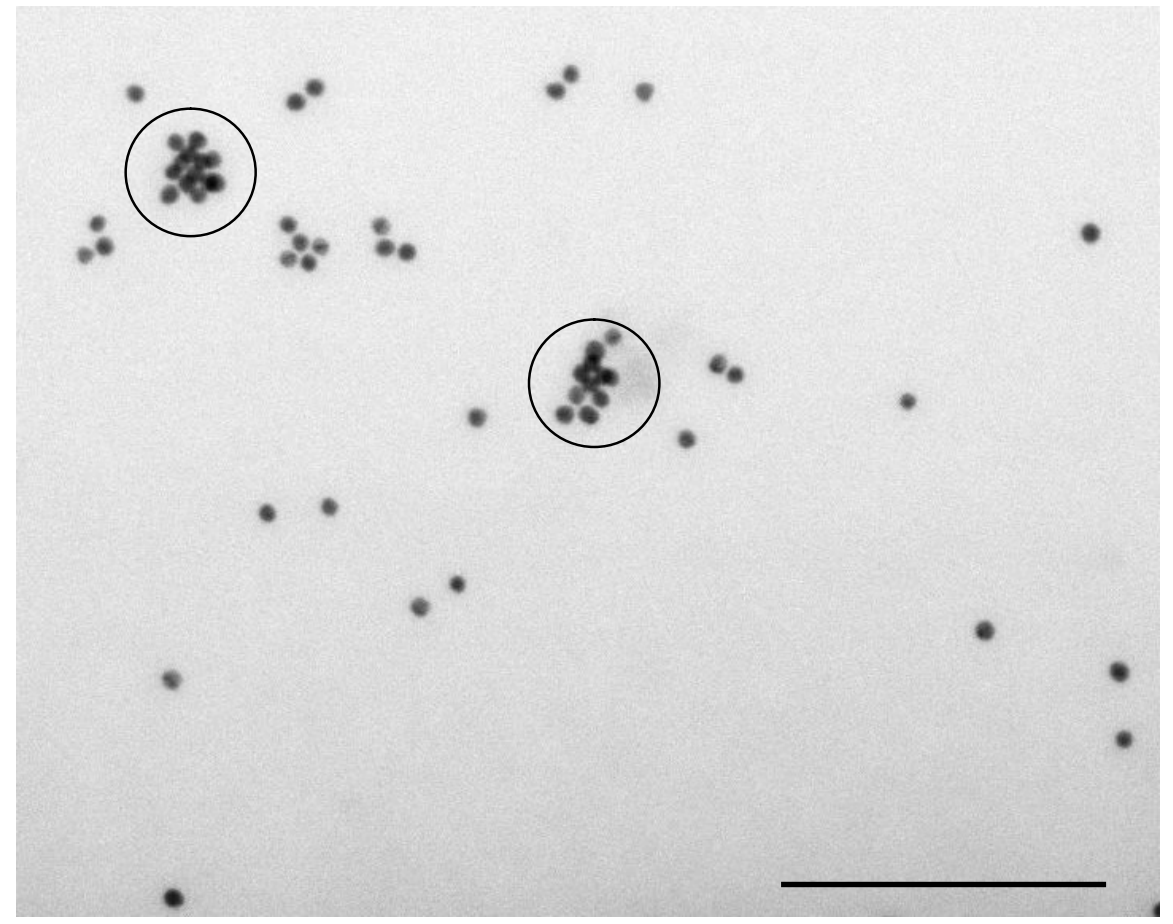

Figure S8c. Example of large area HRSTEM picture of the twelve $10 \mathrm{~nm}$ AuNP helical assembly $\mathrm{BT}_{1,2} \mathrm{~L}$ (bar=150nm). 


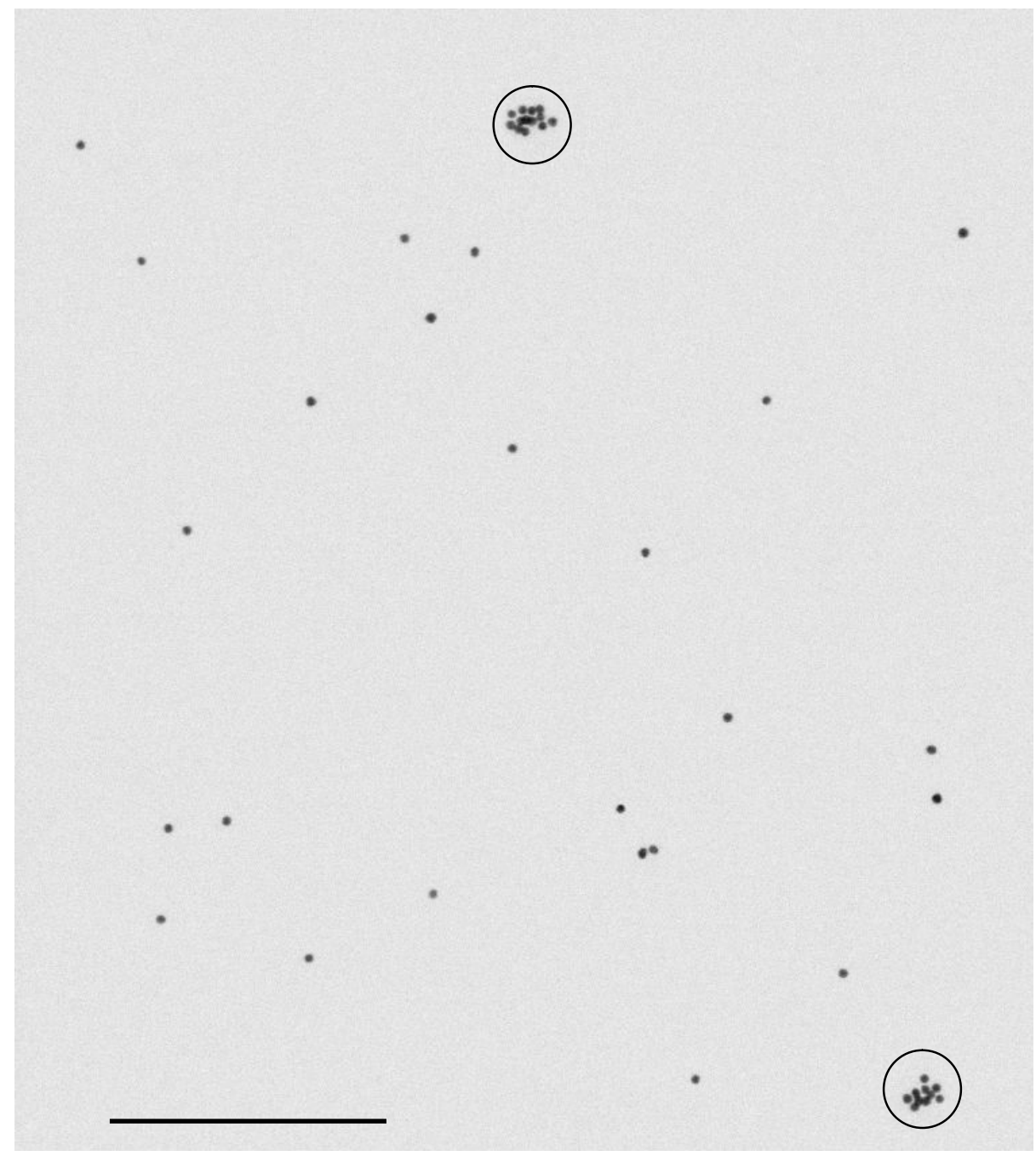

Figure S9a. Example of large area HRSTEM picture of the twelve $10 \mathrm{~nm}$ AuNP helical assembly $\mathrm{BT}_{1,2} \mathrm{R}$ (bar=250nm). 


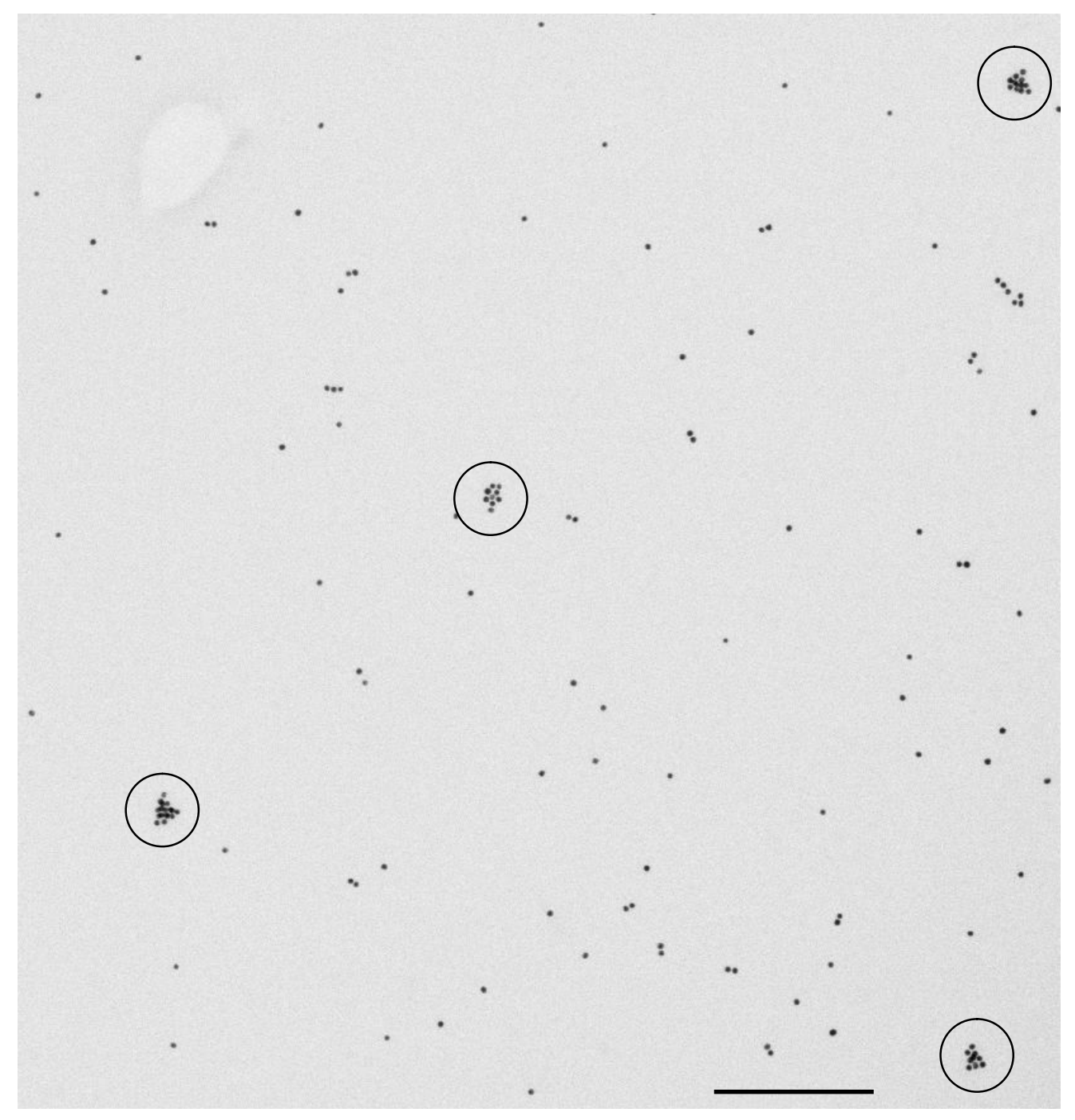

Figure S9b. Example of large area HRSTEM picture of the twelve $10 \mathrm{~nm}$ AuNP helical assembly $\mathrm{BT}_{1,2} \mathrm{R}$ (bar=250nm).

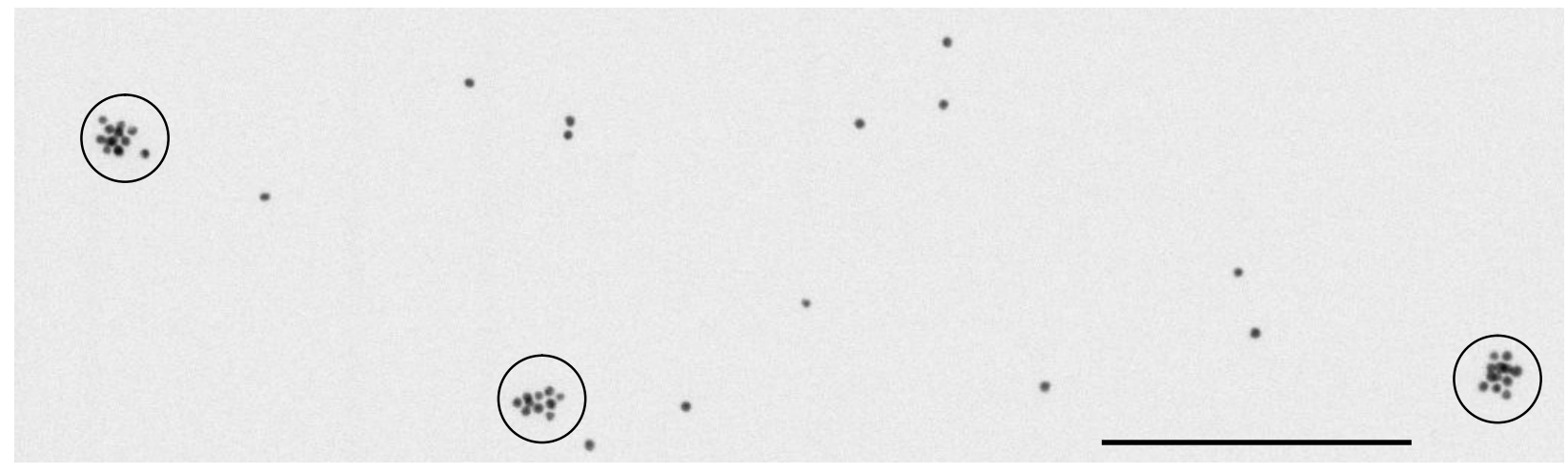

Figure S9c. Example of large area HRSTEM picture of the twelve $10 \mathrm{~nm}$ AuNP helical assembly $\mathrm{BT}_{1,2} \mathrm{R}$ (bar=250nm). 


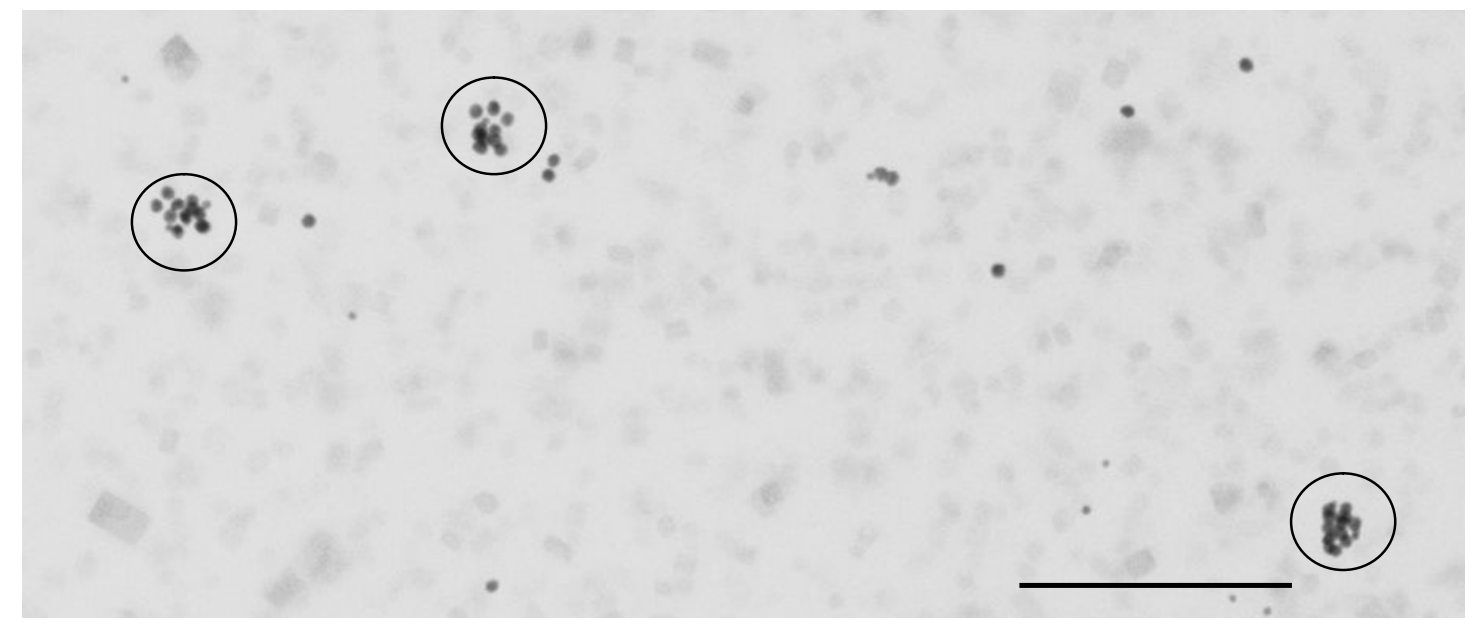

Figure S10a. Example of large area HRSTEM picture of the twelve $10 \mathrm{~nm}$ AuNP helical assembly $\mathrm{BT}_{3,4} \mathrm{R}$ (bar=250nm).

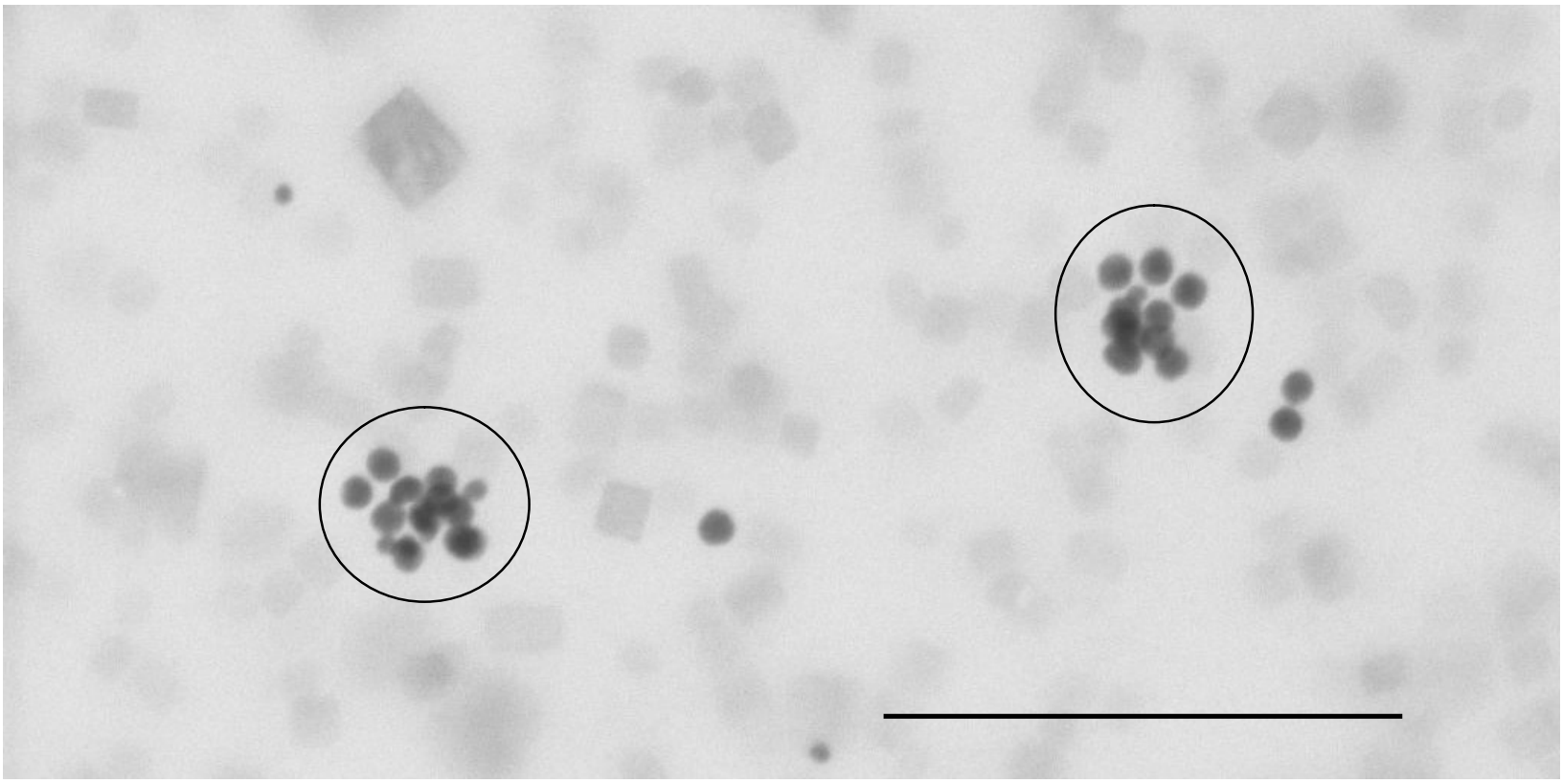

Figure S10b. Example of large area HRSTEM picture of the twelve $10 \mathrm{~nm}$ AuNP helical assembly $\mathrm{BT}_{3,4} \mathrm{R}$ (bar=200nm). 


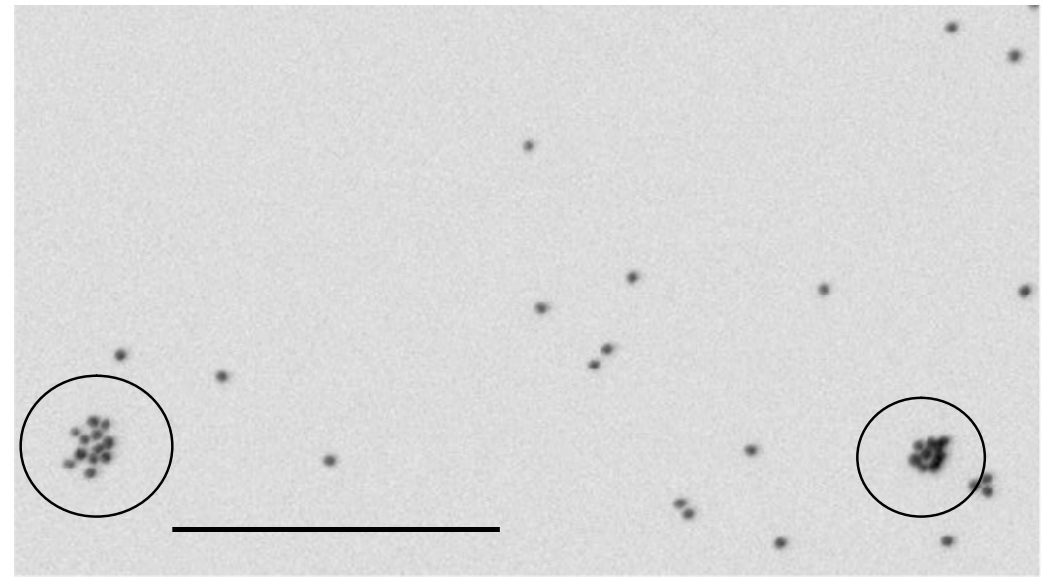

Figure S10c. Example of large area HRSTEM picture of the twelve $10 \mathrm{~nm}$ AuNP helical assembly $\mathrm{BT}_{3,4} \mathrm{R}$ (bar=250nm). 


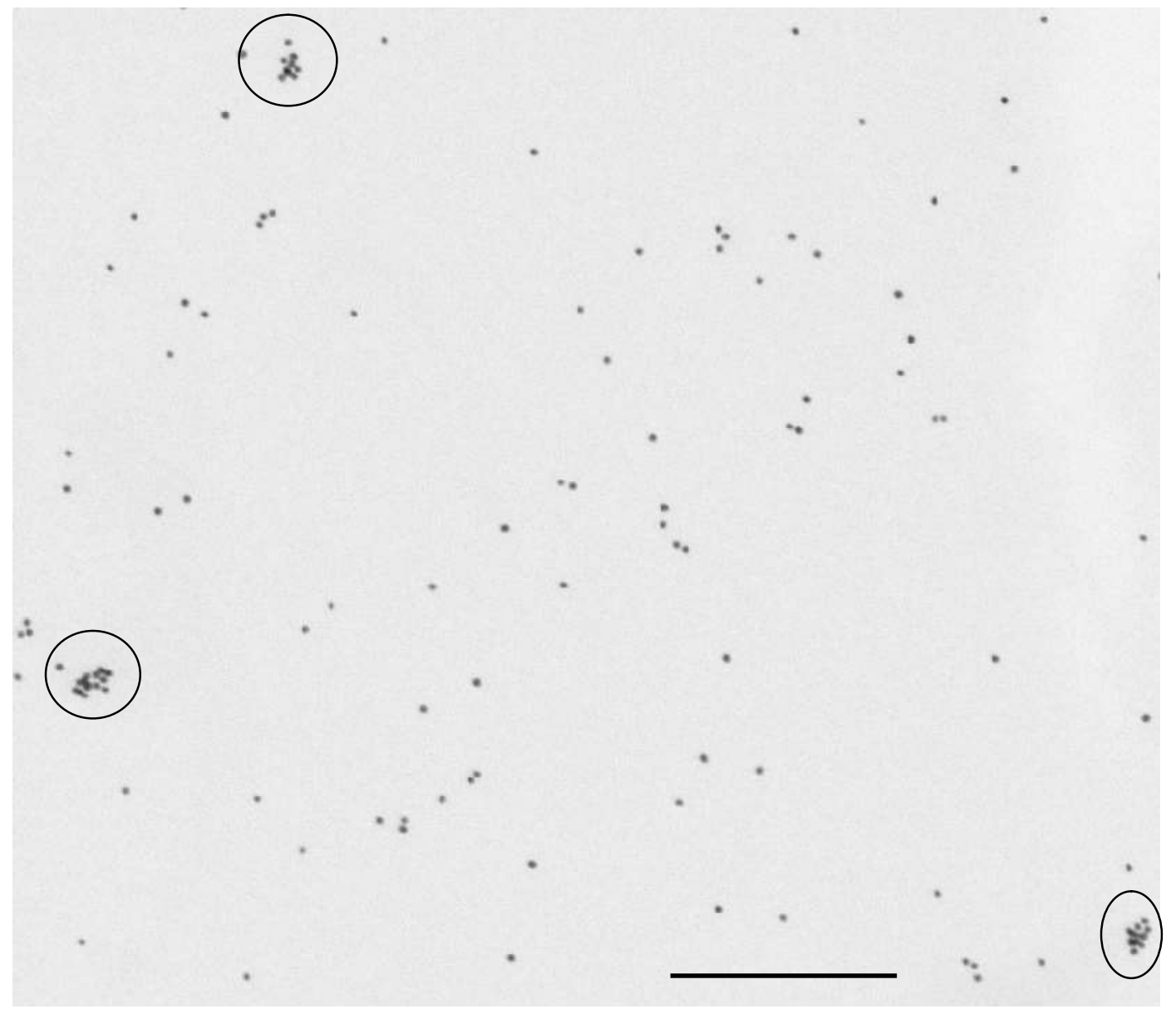

Figure S11a. Example of large area HRSTEM picture of the twelve $10 \mathrm{~nm}$ AuNP helical assembly $\mathrm{BT}_{3,4} \mathrm{~L}(\mathrm{bar}=250 \mathrm{~nm})$. 


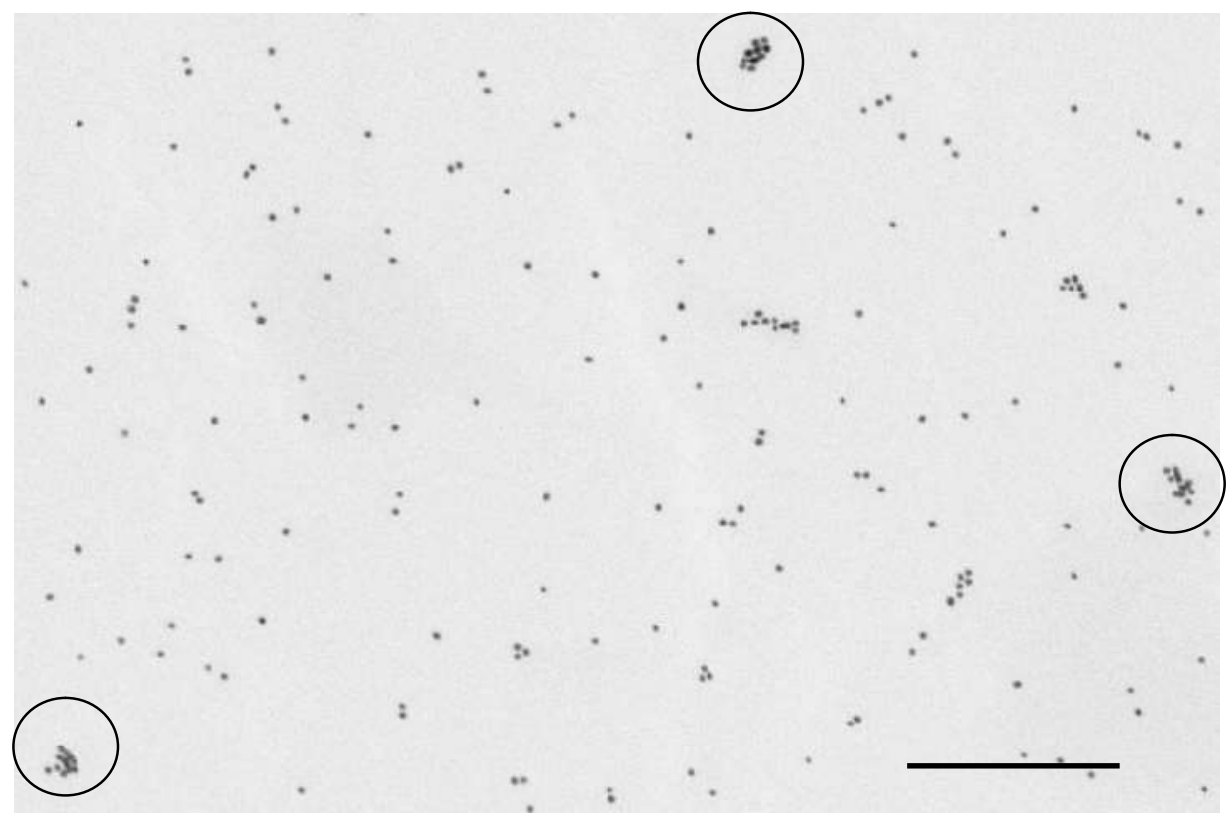

Figure S11b. Example of large area HRSTEM picture of the twelve $10 \mathrm{~nm}$ AuNP helical assembly $\mathrm{BT}_{3,4} \mathrm{~L}$ (bar=250nm). 


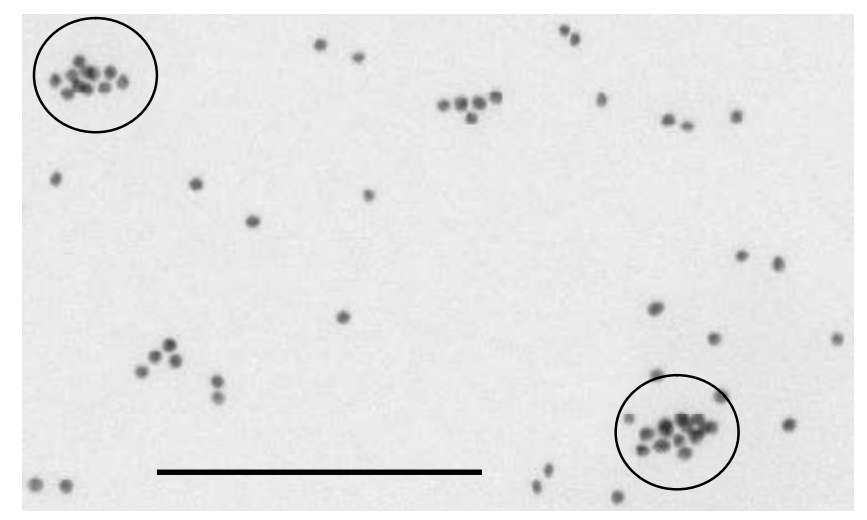

Figure S11c. Example of large area HRSTEM picture of the twelve $10 \mathrm{~nm}$ AuNP helical assembly $\mathrm{BT}_{3,4} \mathrm{~L}($ bar $=200 \mathrm{~nm})$.
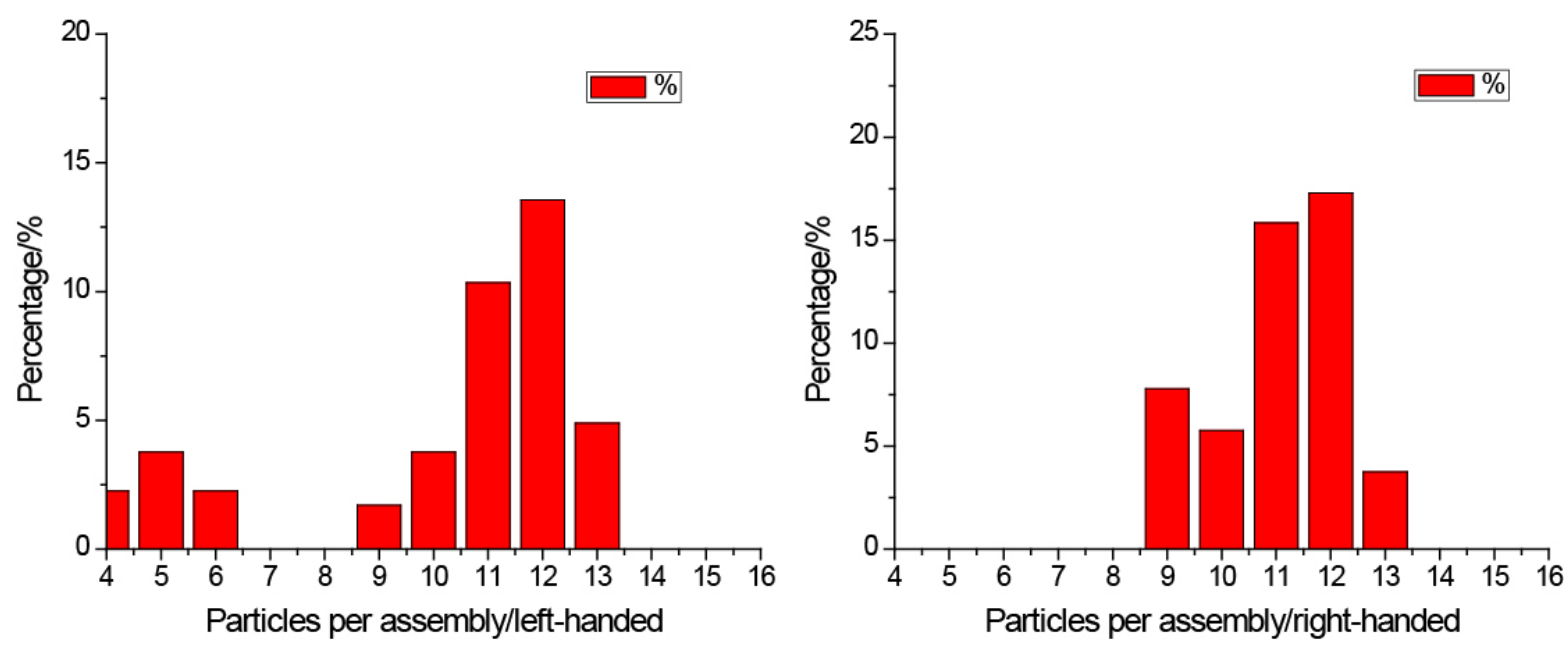

Figure S12. Statistical distribution of the AuNP assemblies containing the respective number of particles. 

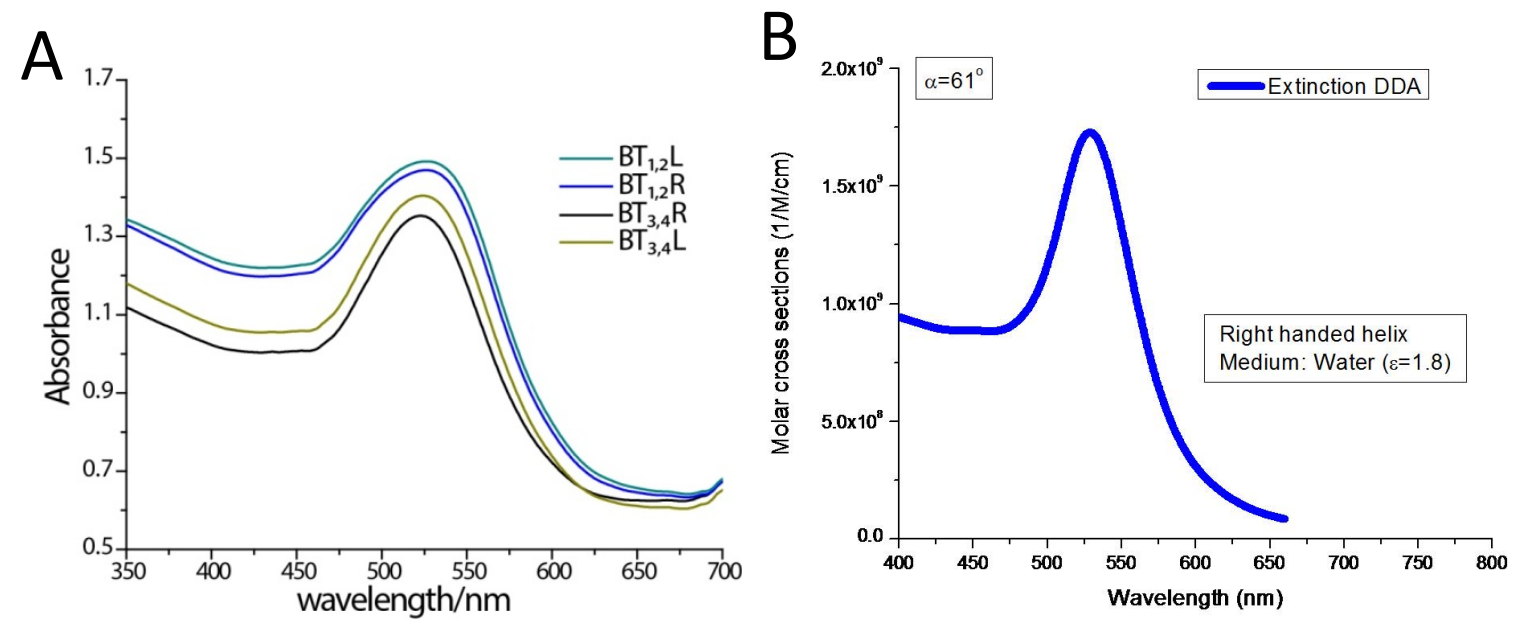

Figure S13. (A) Experimental absorbance spectra of chiroplasmonic structures $B T_{1,2} L, B T_{1,2} R$, $\mathrm{BT}_{3,4} \mathrm{~L}$, and $\mathrm{BT}_{3,4} \mathrm{R}$, respectively. (B) Calculated extintion DDA spectrum of $\mathrm{BT}_{1,2} \mathrm{R}$, used to simulate the CD spectra.
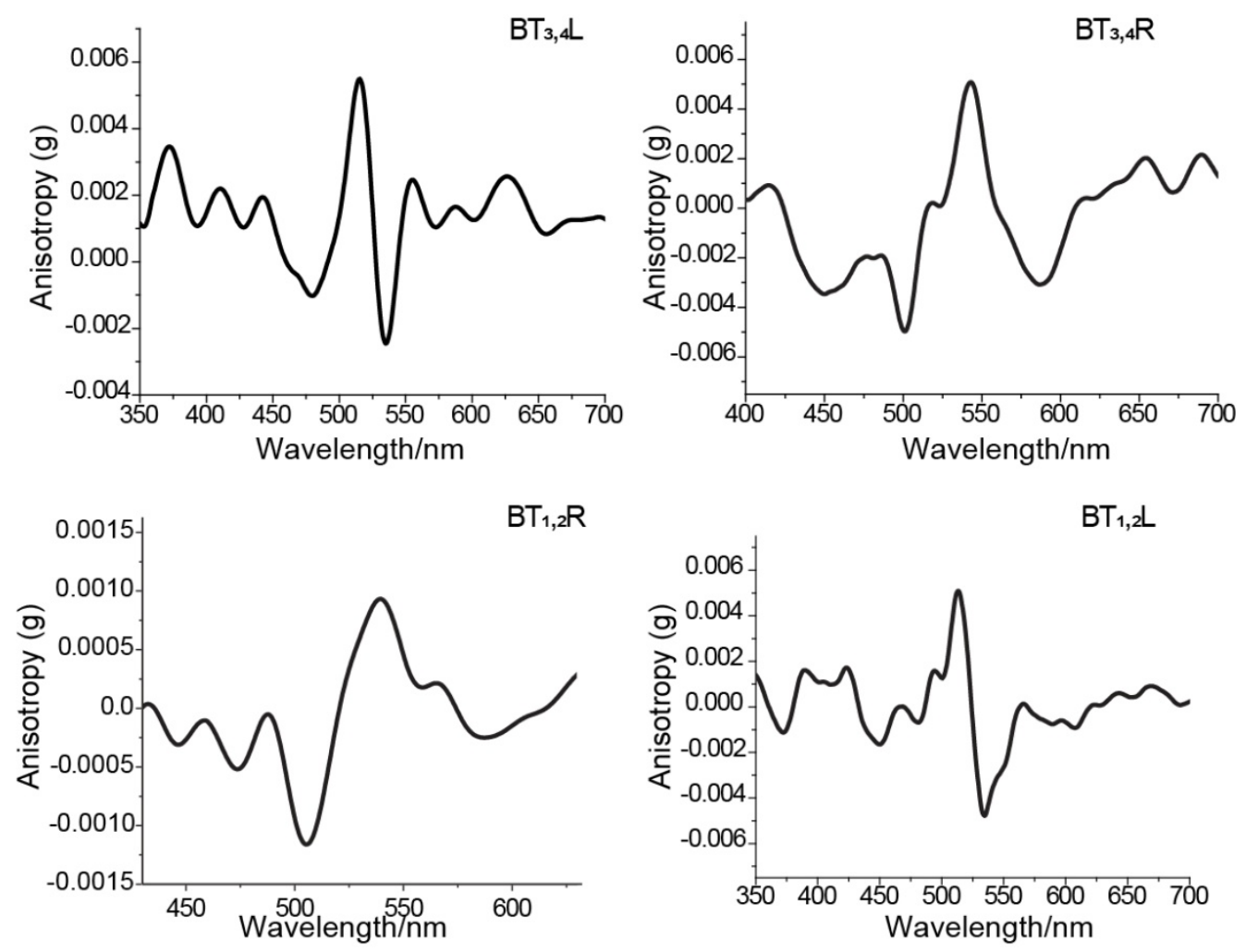

Figure S14. Anisotropy g-factors corresponding to the optical activity of chiroplasmonic structures $\mathrm{BT}_{1,2} \mathrm{~L}, \mathrm{BT}_{1,2} \mathrm{R}, \mathrm{BT}_{3,4} \mathrm{~L}$, and $\mathrm{BT}_{3,4} \mathrm{R}$, respectively. 


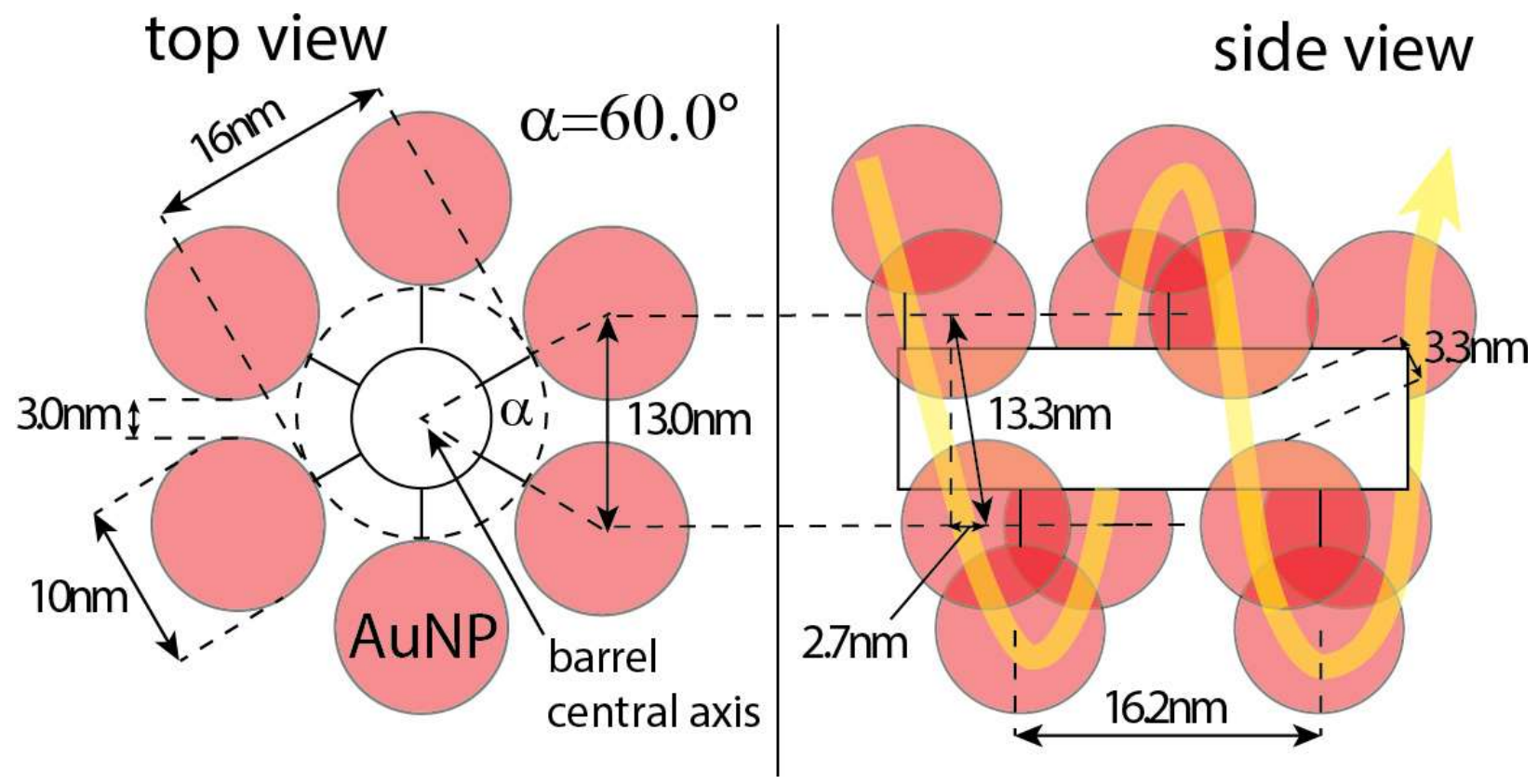

Figure S15. Geometrical model of the right-handed 12 AuNP helical structure decorating the DNA barrel.
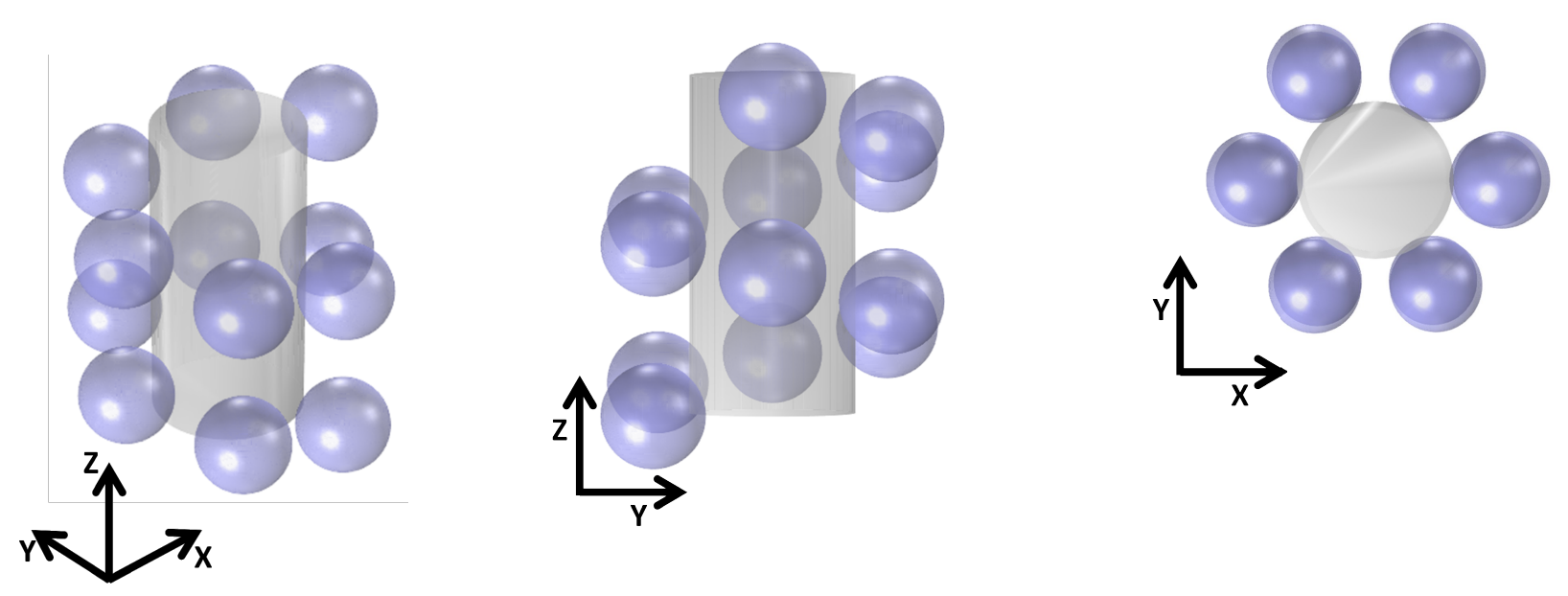

Figure S16a. 3D geometrical model of the left-handed helix for DDA calculations. 

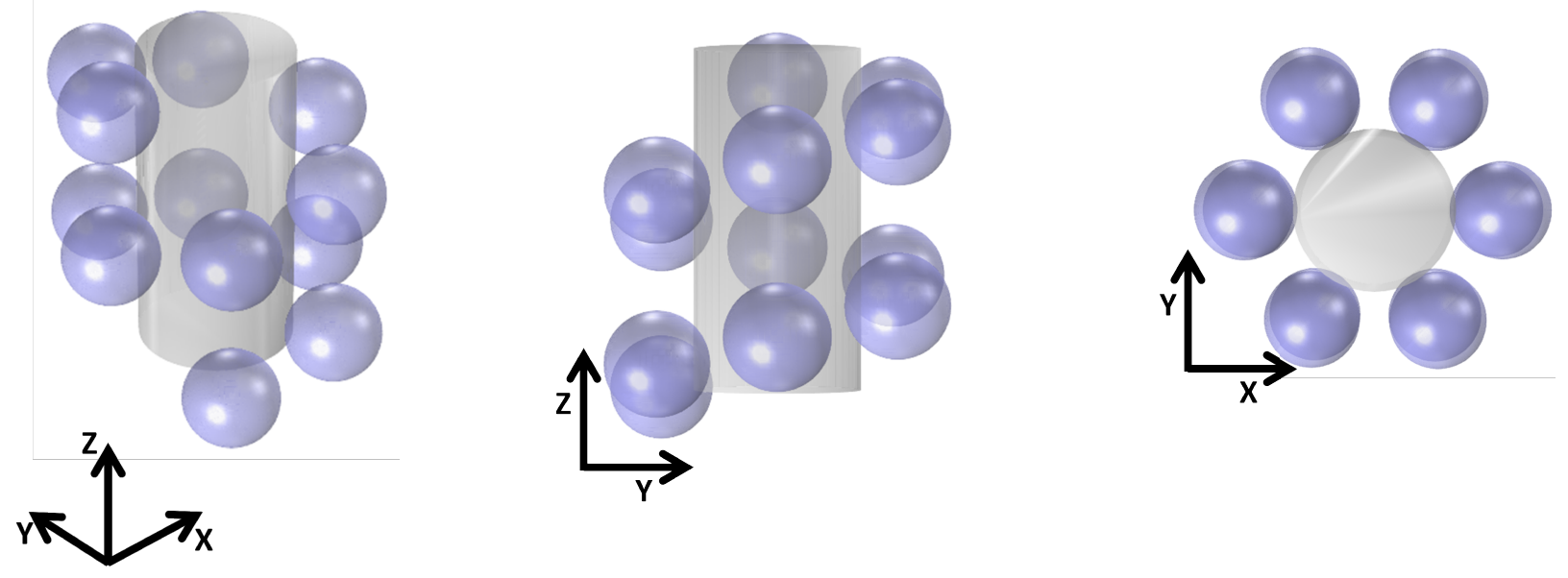

Figure S16b. 3D geometrical model of the right-handed helix for DDA calculations.

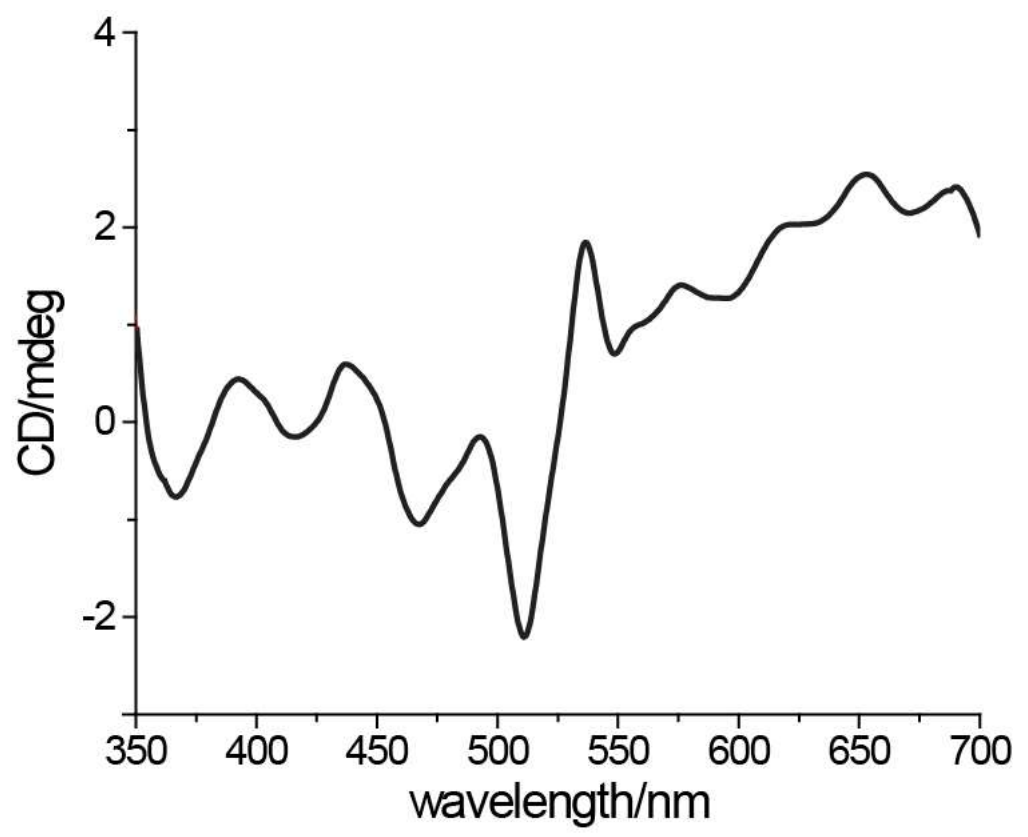

Figure S17. CD spectrum of the chiroplasmonic structure of twelve AuNPs associated with barrel $\mathrm{BT}_{3,4} \mathrm{R}$ where the promoter strands Ps were rendered double stranded by the use of complementary strands PsC. 


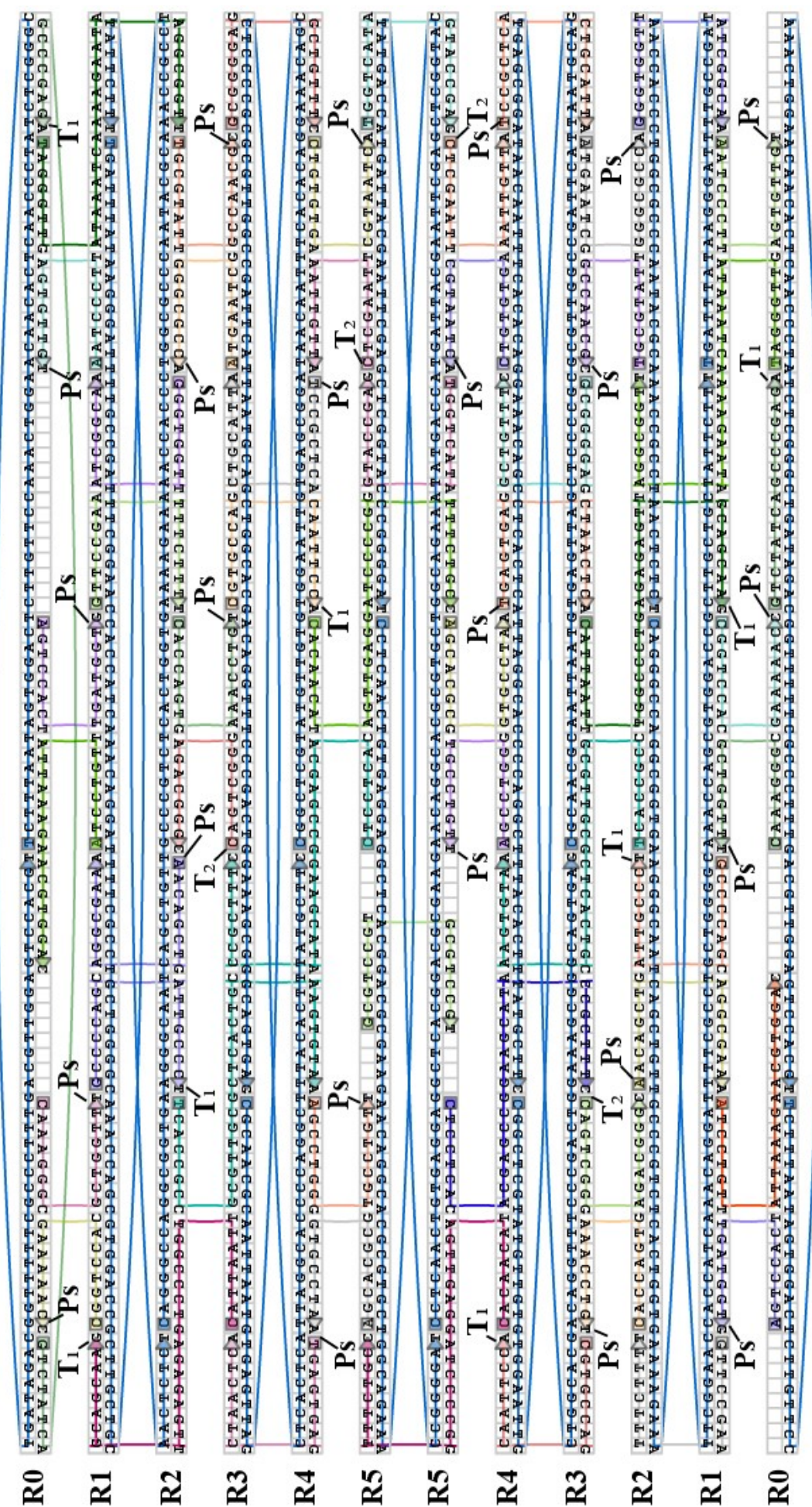

Figure S18. CadNano aided design scheme of the $\mathrm{BT}_{1,2} \mathrm{~L}$ barrel sequences. 


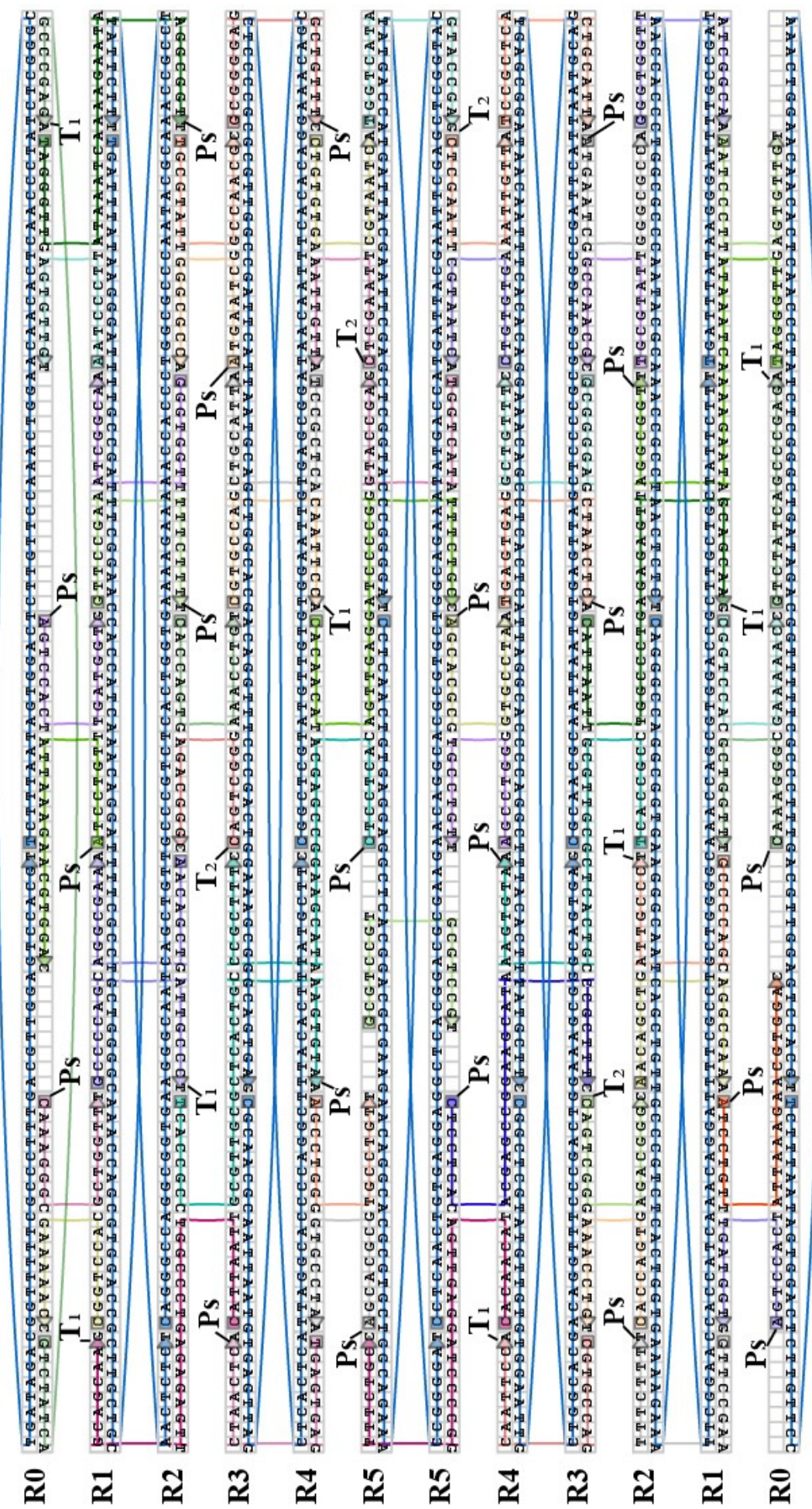

Figure S19. CadNano aided design scheme of the $\mathrm{BT}_{1,2} \mathrm{R}$ barrel sequences. 


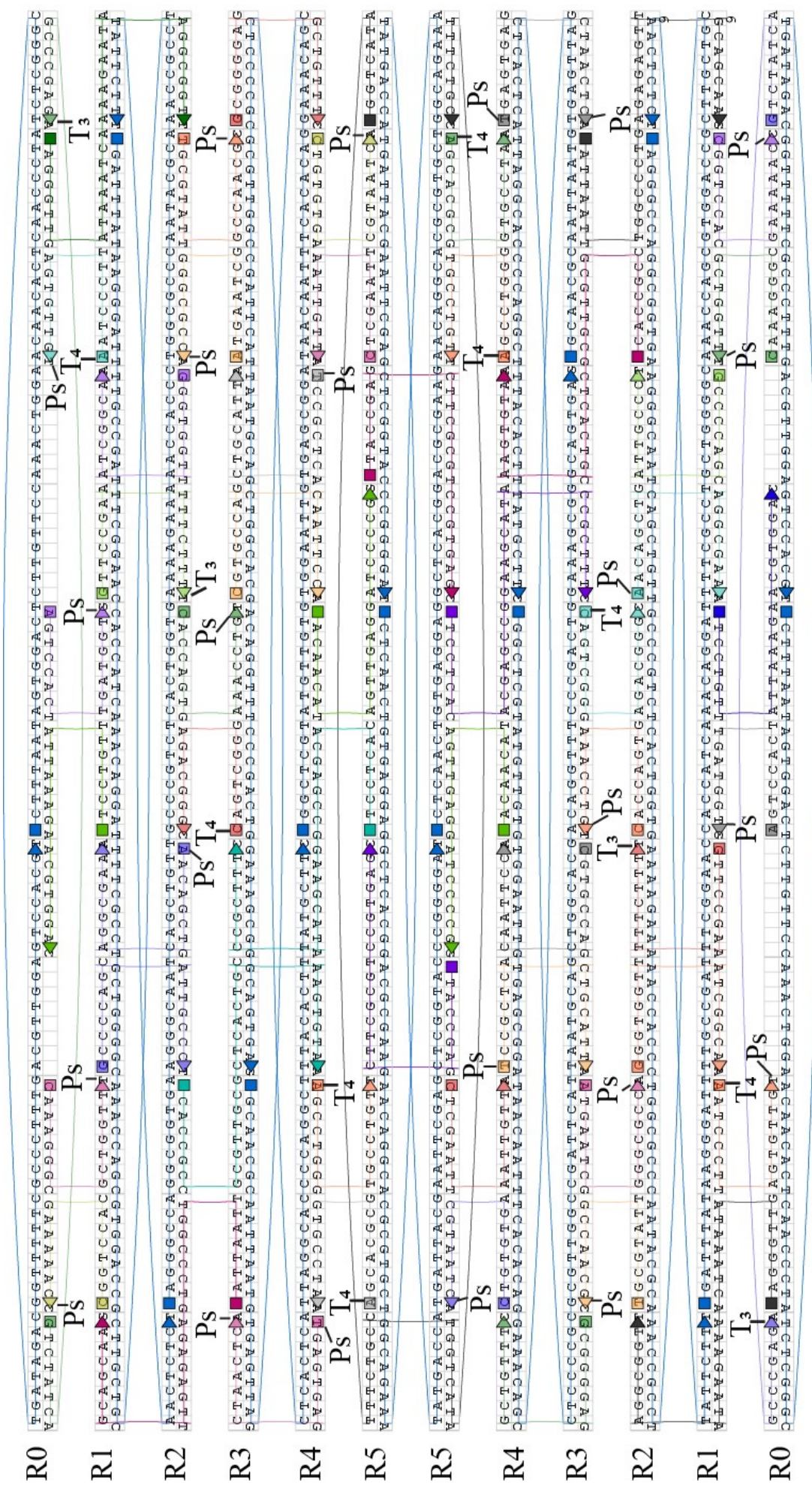

Figure S20. CadNano aided design scheme of the $\mathrm{BT}_{3,4} \mathrm{R}$ barrel sequences. 


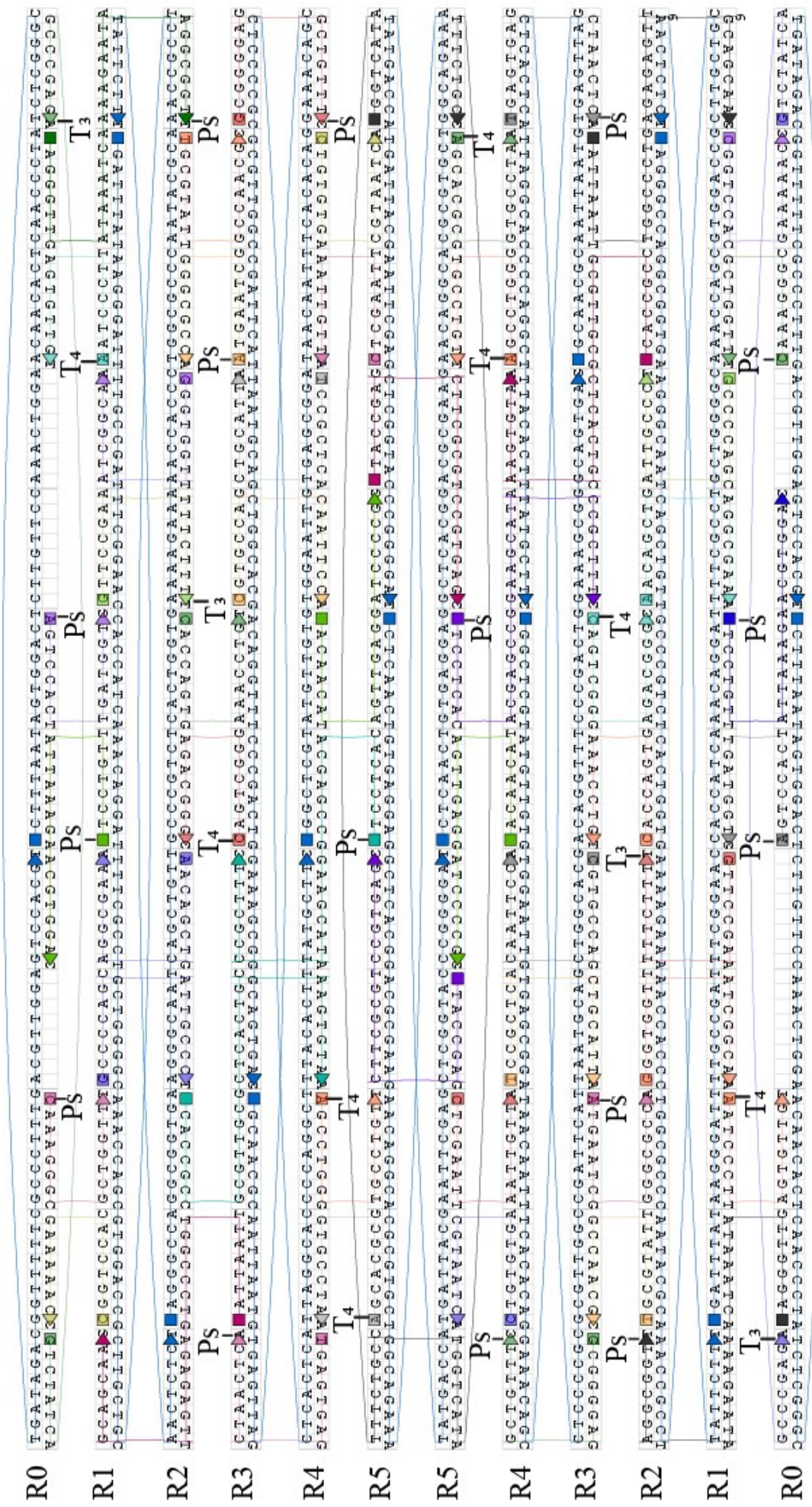

Figure S21. CadNano aided design scheme of the $\mathrm{BT}_{3,4} \mathrm{~L}$ barrel sequences. 


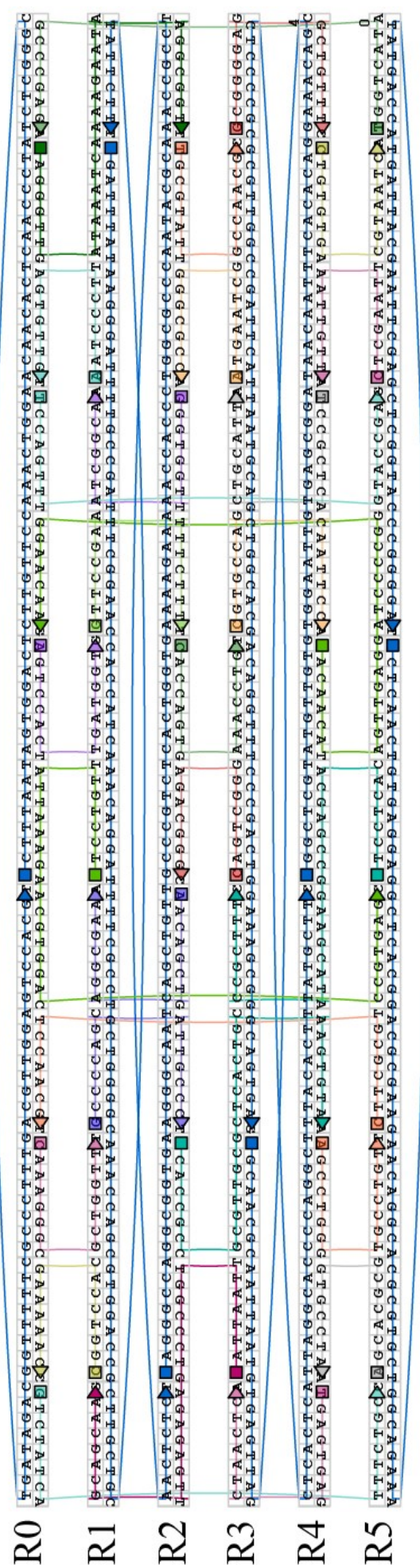

Figure S22. CadNano aided design scheme of the nanotube sequences. 


\begin{tabular}{|c|l|}
\hline R0 & $\begin{array}{l}\text { TCTTTAATAGTGGACTCTTGTTCCAAACTGGAACAACACTCAACCCTATCTCGGGCTGATAGACGGTTT } \\
\text { TTCGCCCTTGACGTTGGAGTCCACGT }\end{array}$ \\
\hline R1 & $\begin{array}{l}\text { TGATTTATAAGGGATTTTGCCGATTTCGGAACCACCATCAAACAGGATTTTCGCCTGCTGGGGCAAACC } \\
\text { AGCGTGGACCGCTTGCTGCTATTCTTT }\end{array}$ \\
\hline R2 & $\begin{array}{l}\text { CAGGGCCAGGCGGTGAAGGGCAATCAGCTGTTGCCCGTCTCACTGGTGAAAAGAAAAACCACCCTGG } \\
\text { CGCCCAATACGCAAACCGCCTAACTCTCT }\end{array}$ \\
\hline R3 & $\begin{array}{l}\text { CGCAACGCAATTAATGTGAGTTAGCTCCCCGCGCTTGGCCGATTCATTAATGCAGCTGGCACGACAG } \\
\text { GTTTCCCGACTGGAAAGCGGGCAGTGAG }\end{array}$ \\
\hline R4 & $\begin{array}{l}\text { CGGCTCGTATGTTGTGTGGAATTGTGAGCGGATAACAATTTCACACAGGAAACAGCCTCACTCATTAG } \\
\text { GCACCCCAGGCTTACACTTAATGCTTC }\end{array}$ \\
\hline R5 & $\begin{array}{l}\text { CCTCAACTGTGAGGAGGCTCACGGACGCGAAGAACAGGCACGCGTGCTGGCAGAAATATGACCATGA } \\
\text { TTACGAATTCGAGCTCGGTACCCGGGGAT }\end{array}$ \\
\hline
\end{tabular}

Table 1. Quasi-ring DNA sequences.

\begin{tabular}{|c|c|}
\hline name & sequence \\
\hline $\mathrm{T}_{1}$ & ATATCTGTGAGCGGC \\
\hline $\mathrm{T}_{2}$ & TCATGCATCTAGACT \\
\hline $\mathrm{T}_{3}$ & ATATCTGTGAGCGGC \\
\hline $\mathrm{T}_{4}$ & TCATGCATCTAGACT \\
\hline $\mathrm{T}_{1,3}{ }^{\prime}$ & thiol-CCG CCG CCG CTC ACA GAT AT \\
\hline$T_{2,4} 4^{\prime}$ & AGT CTA GAT GCA TGA GAT CT-thiol \\
\hline Ps & TTTTTATTTTTATTTTTATTTTTA \\
\hline Stab & thiol-TTTTT \\
\hline $\mathrm{PsC}$ & TAAAAATAAAAATAAAAATAAAAA \\
\hline $\mathrm{T}_{1,3}{ }^{\prime \prime}$ & CGGCGGCGGCGTACTACTACTACTACTACTACTACTACTACATATCTGTGAGCGGCGGCGG \\
\hline $\mathrm{RT}_{1,3}{ }^{\prime \prime}$ & $\begin{array}{l}\text { CCG CCG CCG CTC ACA GAT ATG TAG TAG TAG TAG TAG TAG TAG TAG TAG TAC } \\
\text { GCC GCC GCC G }\end{array}$ \\
\hline $\mathrm{T}_{2,4}{ }^{\prime \prime}$ & AGATCTCATGCATCTAGACTGACTGTCGACACATGCACAGTAGGCAATACCTATACTTATT \\
\hline $\mathrm{RT}_{2,4}{ }^{\prime \prime}$ & $\begin{array}{l}\text { AAT AAG TAT AGG TAT TGC CTA CTG TGC ATG TGT CGA CAG TCA GTC TAG ATG CAT } \\
\text { GAG ATC T }\end{array}$ \\
\hline
\end{tabular}

Table 2. Tethers (T), promoter strands (Ps), and thiolated strands sequences $\left(T_{1,3}{ }^{\prime}\right.$ and $T_{2,4}$ are the thiolated sequences for the surface modification of the AuNPs, and they are complementary to $T_{1}, T_{2}$ and $T_{3}, T_{4}$, respectively; Stab is the short thiolated strand for the stabilization to high salt concentrations of the AuNPs); PsC sequence corresponds to the nucleic acid strand complementary to the Ps strands. All sequences are listed in the $5^{\prime} \rightarrow 3^{\prime}$ orientation. Sequences $\mathrm{T}_{1,3}$ "/ $\mathrm{RT}_{1,3}$ " and $\mathrm{T}_{2,4}$ "/ $\mathrm{RT}_{2,4}$ " are the DNA strands used to elongate/release the tethers $\mathrm{T}^{\prime}$ during the preparation of the single-nucleic acid modified AuNPs. 


\begin{tabular}{|c|c|c|}
\hline Start & End & Sequence \\
\hline $2[55]$ & $3[55]$ & CACCAGTGAAACCTGT-PS \\
\hline $0[55]$ & $1[55]$ & AGTCCACTTGATGGTG-PS \\
\hline $3[72]$ & $2[72]$ & ATGAATCGGGGCGCCA-Ps \\
\hline $1[40]$ & $0[32]$ & ATCCTGTTATTAAAGAACGTGGAC \\
\hline $5[8]$ & $4[8]$ & AGCACGCGGTGCCTAA \\
\hline $1[72]$ & $0[72]$ & AATCCCTTAGTGTTGT-Ps \\
\hline $4[55]$ & $5[56]$ & CACAACATAGTTGAGGATCCCCGGTTTCTGCC \\
\hline $5[88]$ & $5[88]$ & TGGTCATAGTACCGAG \\
\hline $3[88]$ & $4[88]$ & GCGGGGAGGCTGTTTC \\
\hline $5[40]$ & $3[39]$ & СTCCTCACACGAGCCGGAAGCATACCGCTTTC \\
\hline $1[56]$ & $2[56]$ & GTTCCGAATTTCTTTT \\
\hline $5[28]$ & $5[28]$ & GCGTCCGTGCGTCCGT \\
\hline $0[23]$ & $1[23]$ & CAAAGGGCGCTGGTTT-Ps \\
\hline $5[72]$ & $4[72]$ & $\mathrm{T}_{2}$-CTCGAATTAATTGTTA \\
\hline $3[40]$ & $2[40]$ & $\mathrm{T}_{2}$-CAGTCGGGAGACGGGC \\
\hline $3[8]$ & $1[7]$ & CATTAATTTGGCCCTGAGAGAGTTGCAGCAAG-T 1 \\
\hline $2[71]$ & $1[71]$ & GGGTGGTTATCGGCAA \\
\hline $0[87]$ & $2[88]$ & TAGGGTTGATAAATCAAAAGAATAAGGCGGTT \\
\hline $4[8]$ & $5[7]$ & CACAACATAGTTGAGGATCCCCGGTTTCTGCC \\
\hline $2[87]$ & $3[87]$ & TGCGTATTGCCAACGC-Ps \\
\hline $4[87]$ & $5[87]$ & CTGTGTGACGTAATCA-Ps \\
\hline $4[7]$ & $3[7]$ & Ps-TGAGTGAGCTAACTCA \\
\hline $4[71]$ & $3[71]$ & Ps -TCCGCTCACTGCATTA \\
\hline $4[23]$ & $5[23]$ & AGCCTGGGTGCCTGTT-PS \\
\hline $3[56]$ & $4[56]$ & CGTGCCAGCAATTCCA-T $_{1}$ \\
\hline $1[8]$ & $0[8]$ & CGGTCCACGAAAAACC-Ps \\
\hline $2[23]$ & $4[24]$ & TCACCGCCGCGTTGCGCTCACTGCAAGTGTAA \\
\hline $0[7]$ & $0[88]$ & GTCTATCAGCCCGAGA-T $_{1}$ \\
\hline $1[24]$ & $2[24]$ & GCCCCAGCATTGCCCT-T $_{1}$ \\
\hline $2[39]$ & $1[39]$ & Ps -AACAGCTGAGGCGAAA \\
\hline
\end{tabular}

Table 3. Staple DNA sequences for building $B T_{1,2} L$. Numbers in brackets in the first two columns refer to the $5^{\prime}$ position (start) and the 3 ' position (end) according to the sequence map shown in Figure S10, while the number on the left refers to the quasi-ring identification (position 0 is the first base on the left, in respect to the complementary quasi-ring sequence, while position 95 is the last base on the right, in respect to the complementary quasi ring sequence; positions regarding the staples hybridizing to the bottom six quasi-ring sequences refer to position 0 to the right and position 95 to the left, in respect to the complementary sequences of the quasi-rings). 


\begin{tabular}{|c|c|c|}
\hline Start & End & Sequence \\
\hline $2[55]$ & $3[55]$ & CACCAGTGAAACCTGT \\
\hline $0[55]$ & $1[55]$ & Ps-AGTCCACTTGATGGTG \\
\hline $3[72]$ & $2[72]$ & Ps-ATGAATCGGGGCGCCA \\
\hline $1[40]$ & $0[32]$ & Ps-ATCCTGTTATTAAAGAACGTGGAC \\
\hline $5[8]$ & $4[8]$ & Ps-AGCACGCGGTGCCTAA \\
\hline $1[72]$ & $0[72]$ & AATCCCTTAGTGTTGT \\
\hline $4[55]$ & $5[56]$ & CACAACATAGTTGAGGATCCCCGGTTTCTGCC \\
\hline $5[88]$ & $6[88]$ & TGGTCATAGTACCGAG \\
\hline $3[88]$ & $4[88]$ & GCGGGGAGGCTGTTTC-Ps \\
\hline $5[40]$ & $3[39]$ & Ps-CTCCTCACACGAGCCGGAAGCATACCGCTTTC \\
\hline $1[56]$ & $2[56]$ & GTTCCGAATTTCTTTTI-PS \\
\hline $5[28]$ & $5[28]$ & GCGTCCGTGCGTCCGT \\
\hline $0[23]$ & $1[23]$ & Ps-CAAAGGGCGCTGGTTT \\
\hline $5[72]$ & $4[72]$ & $\mathrm{T}_{2}$-CTCGAATTAATTGTTA \\
\hline $3[40]$ & $2[40]$ & $\mathrm{T}_{2}$-CAGTCGGGAGACGGGC \\
\hline $3[8]$ & $1[7]$ & CATTAATTTGGCCCTGAGAGAGTTGCAGCAAG-T 1 \\
\hline $2[71]$ & $1[71]$ & GGGTGGTTATCGGCAA \\
\hline $0[87]$ & $2[88]$ & TAGGGTTGATAAATCAAAAGAATAAGGCGGTT-Ps \\
\hline $4[8]$ & $5[7]$ & CACAACATAGTTGAGGATCCCCGGTTTCTGCC \\
\hline $2[87]$ & $3[87]$ & TGCGTATTGCCAACGC \\
\hline $4[87]$ & $5[87]$ & CTGTGTGACGTAATCA \\
\hline $4[7]$ & $3[7]$ & TGAGTGAGCTAACTCA-Ps \\
\hline $4[71]$ & $3[71]$ & TCCGCTCACTGCATTA \\
\hline $4[23]$ & $5[23]$ & AGCCTGGGTGCCTGTT \\
\hline $3[56]$ & $4[56]$ & CGTGCCAGCAATTCCA-T $_{1}$ \\
\hline $1[8]$ & $0[8]$ & CGGTCCACGAAAAACC \\
\hline $2[23]$ & $4[24]$ & TCACCGCCGCGTTGCGCTCACTGCAAGTGTAA-Ps \\
\hline $0[7]$ & $0[88]$ & GTCTATCAGCCCGAGA-T 1 \\
\hline $1[24]$ & $2[24]$ & GCCCCAGCATTGCCCT-T $_{1}$ \\
\hline $2[39]$ & $1[39]$ & AACAGCTGAGGCGAAA \\
\hline
\end{tabular}

Table 4. Staple DNA sequences for building $B T_{1,2} R$. Numbers in the first two columns refer to the 5 ' position (start) and the 3 ' position (end) according to the sequence map shown in Figure $\mathrm{S} 11$, while the number on the left refers to the quasi-ring identification (position 0 is the first base on the left, in respect to the complementary quasi-ring sequence, while position 95 is the last base on the right, in respect to the complementary quasi ring sequence; positions regarding the staples hybridizing to the bottom six quasi-ring sequences refer to position 0 to the right, and position 95 to the left, in respect to the complementary sequences of the quasi-rings). 


\begin{tabular}{|l|l|l|}
\hline Start & End & Sequence \\
\hline $2[23]$ & $4[24]$ & TCACCGCCGCGTTGCGCTCACTGCAAGTGTAA \\
\hline $0[55]$ & $1[55]$ & AGTCCACTTGATGGTG-Ps \\
\hline $3[8]$ & $1[7]$ & CATTAATTTGGCCCTGAGAGAGTTGCAGCAAG \\
\hline $1[40]$ & $0[32]$ & ATCCTGTTATTAAAGAACGTGGAC \\
\hline $5[8]$ & $4[8]$ & T$_{4}$-AGCACGCGGTGCCTAA \\
\hline $1[56]$ & $2[56]$ & GTTCCGAATTCTTTT-T 3 \\
\hline $3[88]$ & $4[88]$ & GCGGGGAGGCTGTTTC \\
\hline $2[71]$ & $1[71]$ & GGGTGGTTATCGGCAA \\
\hline $5[72]$ & $4[72]$ & CTCGAATTAATTGTTA \\
\hline $2[55]$ & $3[55]$ & CACCAGTGAAACCTGT-Ps \\
\hline $4[23]$ & $5[23]$ & T-AGCCTGGGTGCCTGTT \\
\hline $5[31]$ & $5[39]$ & GTACCGAGCTTCGCGTCCGTGAGC \\
\hline $2[39]$ & $1[39]$ & Ps-AACAGCTGAGGCGAAA \\
\hline $0[87]$ & $2[88]$ & TAGGGTTGATAAATCAAAAGAATAAGGCGGTT \\
\hline $1[72]$ & $0[72]$ & T-AATCCCTTAGTGTTGT-Ps \\
\hline $4[55]$ & $5[63]$ & CACAACATAGTTGAGGATCCCCGG \\
\hline $2[87]$ & $3[87]$ & TGCGTATTGCCAACGC-Ps \\
\hline $5[88]$ & $5[88]$ & TGGTCATATTTCTGCCTGGTCATATTTCTGCC \\
\hline $4[7]$ & $3[7]$ & Ps-TGAGTGAGCTAACTCA-Ps \\
\hline $3[40]$ & $2[40]$ & T-CAGTCGGGAGACGGGC \\
\hline $3[56]$ & $4[56]$ & CGTGCCAGCAATTCCA \\
\hline $5[64]$ & $5[56]$ & GTACCGAGCTTCGCGTCCGTGAGC \\
\hline $0[7]$ & $0[88]$ & GTCTATCAGCCCGAGA-T 3 \\
\hline $5[40]$ & $3[39]$ & CTCCTCACACGAGCCGGAAGCATACCGCTTTC \\
\hline $4[71]$ & $3[71]$ & Ps-TCCGCTCACTGCATTA \\
\hline $1[24]$ & $2[24]$ & GCCCCAGCATTGCCCT \\
\hline $3[72]$ & $2[72]$ & ATGAATCGGGGCGCCA-Ps \\
\hline $0[23]$ & $1[23]$ & CAAAGGGCGCTGGTTT-Ps \\
\hline $4[87]$ & $5[87]$ & CTGTGTGACGTAATCA-Ps \\
\hline $1[8]$ & $0[8]$ & CGGTCCACGAAAAACC-Ps \\
\hline 9
\end{tabular}

Table 5. Staple DNA sequences for building $B T_{3,4} R$. Numbers in the first two columns refer to the 5' position (start) and the 3 ' position (end) according to the sequence map shown in Figure $\mathrm{S} 12$, while the number on the left refers to the quasi-ring identification (position 0 is the first base on the left, in respect to the complementary quasi-ring sequence, while position 95 is the last base on the right, in respect to the complementary quasi ring sequence; positions regarding the staples hybridizing to the bottom six quasi-ring sequences refer to position 0 to the right, and position 95 to the left, in respect to the complementary sequences of the quasi-rings). 


\begin{tabular}{|c|c|c|}
\hline Start & End & Sequence \\
\hline $2[23]$ & $4[24]$ & TCACCGCCGCGTTGCGCTCACTGCAAGTGTAA \\
\hline $0[55]$ & $1[55]$ & Ps-AGTCCACTTGATGGTG \\
\hline $3[8]$ & $1[7]$ & CATTAATTTGGCCCTGAGAGAGTTGCAGCAAG \\
\hline $1[40]$ & $0[32]$ & Ps-ATCCTGTTATTAAAGAACGTGGAC \\
\hline $5[8]$ & $4[8]$ & $\mathrm{T}_{4}$-AGCACGCGGTGCCTAA \\
\hline $1[56]$ & $2[56]$ & GTTCCGAATTTCTTTT-T ${ }_{3}$ \\
\hline $3[88]$ & $4[88]$ & GCGGGGAGGCTGTTTC-Ps \\
\hline $2[71]$ & $1[71]$ & GGGTGGTTATCGGCAA \\
\hline $5[72]$ & $4[72]$ & CTCGAATTAATTGTTA \\
\hline $2[55]$ & $3[55]$ & CACCAGTGAAACCTGT \\
\hline $4[23]$ & $5[23]$ & $\mathrm{T}_{4}$-AGCCTGGGTGCCTGTT \\
\hline $5[31]$ & $5[39]$ & GTACCGAGCTTCGCGTCCGTGAGC \\
\hline $2[39]$ & $1[39]$ & AACAGCTGAGGCGAAA \\
\hline $0[87]$ & $2[88]$ & TAGGGTTGATAAATCAAAAGAATAAGGCGGTT-Ps \\
\hline $1[72]$ & $0[72]$ & $\mathrm{T}_{4}$-AATCCCTTAGTGTTGT \\
\hline $4[55]$ & $5[63]$ & CACAACATAGTTGAGGATCCCCGG \\
\hline $2[87]$ & $3[87]$ & TGCGTATTGCCAACGC \\
\hline $5[88]$ & $5[88]$ & TGGTCATATTTCTGCCTGGTCATATTTCTGCC \\
\hline $4[7]$ & $3[7]$ & TGAGTGAGCTAACTCA-Ps \\
\hline $3[40]$ & $2[40]$ & $\mathrm{T}_{4}$-CAGTCGGGAGACGGGC \\
\hline $3[56]$ & $4[56]$ & CGTGCCAGCAATTCCA \\
\hline $5[64]$ & $5[56]$ & GTACCGAGCTTCGCGTCCGTGAGC \\
\hline $0[7]$ & $0[88]$ & GTCTATCAGCCCGAGA-T 3 \\
\hline $5[40]$ & $3[39]$ & Ps-CTCCTCACACGAGCCGGAAGCATACCGCTTTC \\
\hline $4[71]$ & $3[71]$ & TCCGCTCACTGCATTA \\
\hline $1[24]$ & $2[24]$ & GCCCCAGCATTGCCCT \\
\hline $3[72]$ & $2[72]$ & Ps-ATGAATCGGGGCGCCA \\
\hline $0[23]$ & $1[23]$ & Ps-CAAAGGGCGCTGGTTT \\
\hline $4[87]$ & $5[87]$ & CTGTGTGACGTAATCA \\
\hline $1[8]$ & $0[8]$ & CGGTCCACGAAAAACC \\
\hline
\end{tabular}

Table 6. Staple DNA sequences for building $\mathrm{BT}_{3,4} \mathrm{~L}$. Numbers in the first two columns refer to the 5 ' position (start) and the 3 ' position (end) according to the sequence map shown in Figure $\mathrm{S} 13$, while the number on the left refers to the quasi-ring identification (position 0 is the first base on the left, in respect to the complementary quasi-ring sequence, while position 95 is the last base on the right, in respect to the complementary quasi ring sequence; positions regarding the staples hybridizing to the bottom six quasi-ring sequences refer to position 0 to the right, and position 95 to the left, in respect to the complementary sequences of the quasi-rings). 


\begin{tabular}{|l|l|l|}
\hline Start & End & Sequence \\
\hline $0[55]$ & $1[55]$ & AGTCCACTTGATGGTG \\
\hline $3[88]$ & $4[88]$ & GCGGGGAGGCTGTTTC \\
\hline $1[72]$ & $0[72]$ & AATCCCTTAGTGTTGT \\
\hline $4[55]$ & $0[56]$ & CACAACATAGTTGAGGATCCCCGGGGAACAAG \\
\hline $4[87]$ & $5[87]$ & CTGTGTGACGTAATCA \\
\hline $4[23]$ & $5[23]$ & AGCCTGGGTGCCTGTT \\
\hline $0[7]$ & $5[7]$ & GTCTATCATTTCTGCC \\
\hline $5[40]$ & $3[39]$ & CTCCTCACACGAGCCGGAAGCATACCGCTTTC \\
\hline $1[56]$ & $2[56]$ & GTTCCGAATTTCTTTT \\
\hline $0[23]$ & $1[23]$ & CAAAGGGCGCTGGTTT \\
\hline $5[24]$ & $0[24]$ & CTTCGCGTTCCAACGT \\
\hline $3[40]$ & $2[40]$ & CAGTCGGGAGACGGGC \\
\hline $3[8]$ & $1[7]$ & CATTAATTTGGCCCTGAGAGAGTTGCAGCAAG \\
\hline $3[72]$ & $2[72]$ & ATGAATCGGGGCGCCA \\
\hline $0[87]$ & $2[88]$ & TAGGGTTGATAAATCAAAAGAATAAGGCGGTT \\
\hline $5[8]$ & $4[8]$ & AGCACGCGGTGCCTAA \\
\hline $2[55]$ & $3[55]$ & CACCAGTGAAACCTGT \\
\hline $2[87]$ & $3[87]$ & TGCGTATTGCCAACGC \\
\hline $0[71]$ & $5[71]$ & TCCAGTTTGTACCGAG \\
\hline $4[71]$ & $3[71]$ & TCCGCTCACTGCATTA \\
\hline $4[7]$ & $3[7]$ & TGAGTGAGCTAACTCA \\
\hline $3[56]$ & $4[56]$ & CGTGCCAGCAATTCCA \\
\hline $1[8]$ & $0[8]$ & CGGTCCACGAAAAACC \\
\hline $2[23]$ & $4[24]$ & TCACCGCCGCGTTGCGCTCACTGCAAGTGTAA \\
\hline $1[24]$ & $2[24]$ & GCCCCAGCATTGCCCT \\
\hline $1[40]$ & $5[39]$ & ATCCTGTTATTAAAGAACGTGGACCCGTGAGC \\
\hline $2[39]$ & $1[39]$ & AACAGCTGAGGCGAAA \\
\hline $2[71]$ & $1[71]$ & GGGTGGTTATCGGCAA \\
\hline $5[72]$ & $4[72]$ & CTCGAATTAATTGTTA \\
\hline $5[88]$ & $0[88]$ & TGGTCATAGCCCGAGA \\
\hline
\end{tabular}

Table 7. Staple DNA sequences for building the nanotubes. Numbers in the first two columns refer to the $5^{\prime}$ position (start) and the 3 ' position (end) according to the sequence map shown in Figure S14, while the number on the left refers to the quasi-ring identification (position 0 is the first base on the left, in respect to the complementary quasi-ring sequence, while position 95 is the last base on the right, in respect to the complementary quasi ring sequence). 


\section{REFERENCES}

(1) Zheng, H.; Xiao, M.; Yan, Q.; Ma, Y.; Xiao, S.-J. J. Am. Chem. Soc. 2014, 136, 10194.

(2) Elbaz, J.; Cecconello, A.; Fan, Z.; Govorov, A. O.; Willner, I. Nat. Commun. 2013, 4:2000.

(3) Draine, B. T.; Flatau, P. J. J. Opt. Soc. Am. 1994, 11, 1491.

(4) Draine, B.T.; Flatau, P. J. J. Opt. Soc. Am. A 2008, 25, 2593.

(5) Flatau, P. J.; Draine, B. T. Optics Express 2012, 20, 1247.

(6) Johnson, P. B.; Christy, R. W. Phys. Rev. B. 1972, 6, 4370.

(7) Fan, Z.; Govorov, A. O. Nano Lett. 2012, 12, 3283. 